\title{
CUESTIONARIO DE OPCIONES MÚLTIPLES PARA EVALUAR CREENCIAS SOBRE EL APRENDIZAJE DE LAS CIENCIAS
}

\author{
Marín, Nicolás ${ }^{1}$ y Benarroch, Alicia ${ }^{2}$ \\ ${ }^{1}$ Departamento de Didáctica de las Matemáticas y de las Ciencias Experimentales. Universidad de Almería \\ ${ }^{2}$ Departamento de Didáctica de las Ciencias Experimentales. Universidad de Granada \\ nicolas.marin@gmail.com \\ aliciabb@ugr.es
}

\begin{abstract}
Resumen. En este trabajo se muestra el diseño de un cuestionario de opciones múltiples (COMVdA) para evaluar las creencias relacionadas con el conocimiento y el aprendizaje del alumno de ciencias en el contexto de la formación de futuros profesores de secundaria. Se ha tratado de mantener un principio de máxima coherencia con los criterios teóricos y estructurales utilizados previamente en el diseño, desarrollo y evaluación de un cuestionario previo sobre la visión de las ciencias (COMVdC).

La característica más destacada del proceso seguido es que ha posibilitado la discriminación de ítems de baja calidad frente a otros de media y alta, permitiendo por tanto su autocorrección. Se discute la validez y la fiabilidad del cuestionario en el estado que se presenta y se extraen conclusiones referidas a sus resultados y sus posibilidades para ser introducido como herramienta tanto de evaluación como de enseñanza en la formación de futuros profesores de ciencias.
\end{abstract}

Palabras clave. Creencias sobre el aprendizaje, formación de profesores, cuestionario de opciones múltiples.

\section{Questionnaire of multiple-choice to assessment beliefs about learning of science}

Summary. This paper shows the design of a multiple-choice questionnaire (COMVdA) to assess the beliefs related to science learning in the context of the training of future teachers of secondary schools. It has tried to maintain a principle of maximum coherence with the theoretical and structural criteria. These criteria have been previously used in the design, development and evaluation of a previous questionnaire about the vision of science (COMVdC).

The striking feature of the process is that it has made possible the discrimination of items of low quality compared to others of medium and high quality, becoming therefore a process of self-correction. We discuss the validity and reliability of the questionnaire in the state that is presented and draw we conclusions regarding their findings and their potential to be introduced as a tool for both evaluation and instruction in the training of future science teachers.

Keywords. Beliefs on the learning, training, questionnaire multiple-choice questionnaire.

\section{INTRODUCCIÓN}

En los procesos de enseñanza y aprendizaje siempre aparece una constante: un alumno que debe adquirir nuevos contenidos. De hecho, de los tres elementos básicos que conforman el triángulo didáctico, a saber, alumno, profesor y contenido, el primero y el último son insustituibles. Sin embargo, en ausencia del profesor y presencia, por ejemplo, de materiales didácticos apropiados, puede seguir teniendo lugar el proceso de enseñanza-aprendizaje.

El estudiante frente a un contenido que debe aprender constituye el esquema más básico de los procesos de enseñanza-aprendizaje. Se puede enseñar de forma ex- celente, pero será la motivación del estudiante, sus capacidades intelectuales, sus destrezas y habilidades de estudio, sus expectativas, etc., los factores más determinantes en el éxito del proceso.

Distintos autores (Hollon y Anderson, 1987; Porlán, 1989; Prawat, 1992; Hewson y Hollon, 1994; López, 1995) ponen de manifiesto la importancia que tienen las concepciones de los profesores sobre el aprendizaje. En este sentido, se situarían creencias del profesorado como (Sanmartí, 2002): 
- Los alumnos aprenden si están atentos a la explicación del profesor y estudian. Si no lo hacen, es porque estudian poco o tienen problemas familiares.

- Ese aprendizaje se manifiesta como lo que son capaces de explicar en los exámenes.

- Hay que cambiar las ideas de los alumnos por la «verdad científica».

En el contexto de la formación de profesores, se ha abordado el problema de las creencias sobre el aprendizaje desde diferentes direcciones:

- Unos trabajos tratan de precisar la visión predominante con que el profesorado entiende el proceso de enseñanzaaprendizaje de las ciencias. En este sentido, la mayoría de los trabajos con muestras amplias de profesores concluyen que las posiciones de recepción por «transmisión verbal del conocimiento» o de adquisición «por simple actividad de los alumnos» son bastante frecuentes. Por contra, el desarrollo de visiones constructivistas, que son las consideradas de mayor interés educativo, implicaría una evolución paulatina de los docentes y estarían representadas minoritariamente dentro del profesorado (Porlán, Rivero y Martín del Pozo, 1997; Porlán, Rivero y Martín del Pozo, 1998; Porlán y Martín del Pozo, 2004). Sin embargo, otros trabajos de corte más cualitativo muestran que, incluso cuando se manifiestan las concepciones constructivistas, tampoco son tan uniformes; así, en el estudio de cuatro casos realizado por Mellado (1996), se detecta, en todos ellos, diferentes grados de una visión constructivista del aprendizaje.

- Otros centran su atención en las relaciones entre las creencias del profesorado sobre la enseñanza y aprendizaje de las ciencias con su conducta en el aula al enseñar ciencias (Dillon et al., 1994; Lorsbach et al., 1992) encontrando contradicciones entre creencias explícitas y comportamiento. Éste parece más bien matizado por el conjunto de valores, sentimientos, pensamientos y acciones formadas durante sus experiencias como estudiantes (Huibretse, Korthagen y Wubbels, 1994).

- Asimismo, otra línea de trabajo trata de evaluar el grado de coherencia entre las visiones del profesorado sobre aprendizaje y sobre la enseñanza. Estos estudios muestran que los profesores no poseen siempre concepciones uniformes y coherentes sobre la enseñanza y el aprendizaje de sus alumnos (Desaultes, 1993; Hewson, Kerby y Cook, 1995). En concreto, existe mayor coherencia entre enseñanza y aprendizaje cuando se mantienen posiciones más simples y tradicionales que cuando están más cercanas al constructivismo (Baena, 2000; Boulton-Lewis et al., 2001). Evidentes incoherencias se ponen de manifiesto en el estudio realizado por Ruiz et al. (2005), que analizaron la evolución de las concepciones de una profesora de secundaria entre 1993 y 2002 mediante mapas cognitivos elaborados a partir del cuestionario INPECIP (Inventario de Creencias Pedagógicas y Científicas), de Porlán et al. (1997). Encontraron que la profesora experimentaba en estos 9 años una notable evolución de sus concepciones sobre la naturaleza de la ciencia. Sin embargo, con relación a la enseñanza, ya en 1993 era algo constructivista y, en conse- cuencia, la evolución fue menor. Respecto al aprendizaje, ya en 1993 mostraba una tendencia plenamente constructivista.

En los trabajos citados anteriormente, los investigadores, de modo generalizado, admiten la visión constructivista, aun con distintos matices y versiones, como la más adecuada para interpretar el aprendizaje. Esta visión es la misma que se mantiene en el ámbito de la psicología, donde igualmente se intenta delimitar las creencias sobre enseñanza y aprendizaje y su relación con la actuación docente. Así, las diferentes aportaciones que realizan el grupo de Pozo y otros. (2006) arrojan bastante luz en la temática de las creencias sobre el aprendizaje y sus relaciones con la enseñanza, por lo que se van a tener en consideración.

Este trabajo contiene una aportación más al esfuerzo por delimitar las creencias del profesorado sobre enseñanza y aprendizaje. Concretamente se muestra el fundamento, diseño, desarrollo y validación de un nuevo cuestionario que intenta recoger buena parte de los planteamientos de los trabajos citados pero también las consideraciones de las aportaciones realizadas desde la psicología cognitiva que se acaban de citar.

Los fundamentos teóricos de este cuestionario son paralelos y coherentes a los establecidos en un trabajo anterior donde se mostraba el proceso de elaboración y validación de un cuestionario de creencias sobre el conocimiento de ciencias (Marín y Benarroch, 2009). Específicamente, en este trabajo pretendemos:

a) Mostrar los contenidos, temáticas y aspectos ligados al aprendizaje y su relación con la enseñanza (que denominaremos «sistemática de contextos») que podrían ser de interés para futuros profesores de ciencias de secundaria, y que, en consecuencia, se pretenden evaluar con el cuestionario.

b) Describir el proceso y ejemplificar la elaboración inicial de los ítems de opciones múltiples, atendiendo a la sistemática de contextos anteriormente establecida. Se usará la visión constructivista para formular la opción que técnicamente jugará el papel de «correcta» y las posiciones más empiristas, racionalistas, realistas o reduccionistas para precisar los distractores.

c) Mostrar el proceso utilizado en la validación del cuestionario inicial, tras su administración a una muestra de 45 futuros profesores de secundaria en un curso del CAP desarrollado en 2003.

d) Discutir la utilidad y las limitaciones del cuestionario final, tras su administración a una segunda muestra de 50 futuros profesores de secundaria en un curso del CAP desarrollado en 2005.

e) Extraer conclusiones referidas a los resultados del cuestionario y sus posibilidades para ser introducido como herramienta tanto de evaluación como de enseñanza en la formación de futuros profesores de ciencias.

Es importante destacar que el objetivo principal del cuestionario que se trata de construir es que sea representativo de los contenidos que, en relación con el conocimiento del alum- 
no y su aprendizaje, han sido impartidos en la formación de los futuros docentes, a fin de que sus mediciones permitan evaluar adecuadamente el grado de aprendizaje experimentado. Por tanto, no estamos invalidando la utilidad de otros cuestionarios que también evalúan el aprendizaje (como, por ejemplo, el INPECIP antes citado de Porlán et al., 1997) pero que no responden explícitamente al requisito de que sus preguntas guarden una correspondencia previamente establecida con cada uno de los contenidos de enseñanza.

\section{FUNDAMENTOS TEÓRICOS DEL CUESTIO- NARIO DE OPCIONES MÚLTIPLES SOBRE LA VISION DEL APRENDIZAJE DE CIENCIAS (COMVda)}

Para entender la génesis y fundamento del cuestionario de opciones múltiples sobre la visión del aprendizaje (COMVdA) que, en su versión más actual, se muestra en el anexo 1, es necesario aludir a un trabajo anterior realizado por nosotros (Marín y Benarroch, 2009) donde se describe con detalle el diseño, construcción y validación de un cuestionario sobre la visión de ciencias (COMVdC).

Para el cuestionario COMVdC se optaba por el término «visiones de ciencias» (McComas, Clough y Almazroa, 1998; Fernández et al., 2002) y su acrónimo VdC para hacer alusión a los aspectos epistemológicos de la actividad constructiva de este conocimiento. Ahora, en coherencia, para referirnos a las creencias, opiniones y posturas sobre el aprendizaje de las ciencias, su relación con la enseñanza y, en general, a la organización cognitiva y a la construcción del conocimiento del aprendiz de ciencias, usaremos el término «visión del aprendizaje» y su acrónimo $V d A$. Aunque el significante usado es claramente restrictivo respecto a los distintos aspectos cognitivos que se han listado, se ha visto conveniente su uso por estar más consensuado en la literatura.

Aunque es obvio que los contenidos del COMVdA y COMVdC son diferentes, ambos se estructuran bajo el mismo marco epistemológico, del cual se deducen criterios y orientaciones semejantes y coherentes entre sí para el desarrollo de cada uno de los cuestionarios citados.

El marco epistemológico que aquí se adopta, desarrollado con más detalle en una publicación anterior (Marín, 2003b), parte de las posiciones mejor consensuadas en el ámbito de la enseñanza de las ciencias (McComas, Clough y Almazroa, 1998; Fernández et al., 2002; Osborne et al., 2003; Vázquez, Acevedo y Manassero, 2004). Concretamente, cataloga como menos adecuadas para interpretar o evaluar el conocimiento las posiciones extremas del empirismo, racionalismo y los modelos reduccionistas para entender el conocimiento (Peñalver, 1988), mientras que las posiciones constructivistas se consideran más adecuadas (ver Delval, 1997; Marín, 2003b; Scheuer et al., 2006).

Por otro lado, para ajustar y concretar el marco teórico elegido en la confección práctica de ítems de opciones múltiples, se hace preciso optar por una de las dos versiones del constructivismo, mecanicista u organicista, desde las cuales se puede concebir la realidad. Estas versiones han sido discutidas con detalle anteriormente (Pozo et al., 1996; Delval, 1997; Marín, 2003b). Muy brevemente:

- El constructivismo mecanicista es una postura reduccionista que interpreta la realidad usando la metáfora de la máquina. La realidad puede ser analizada y desmontada en las partes que la componen, asumiendo que la suma de las partes es igual al todo (visión de la realidad como colección de sistemas cerrados). Y puede ser explicada conociendo las relaciones de causa-efecto que existe entre las partes, asumiendo que estas relaciones causales son simples y proporcionales (Luffiego, 2001). En esta línea se enmarcan el procesamiento de la información y el conexionismo (Delval, 1997; Pozo, 2003).

- El constructivismo organicista es antirreduccionista al negar que se puedan explicar los distintos fenómenos de la realidad analizando los elementos que lo componen. Esta postura interpreta la realidad como un organismo vivo donde las propiedades del todo no son iguales a la suma de las de cada parte. En consecuencia, acercarse a la comprensión de un fenómeno supone estudiar dialécticamente el proceso de su evolución. El contexto de confrontación dialéctica entre partes, así como la propia delimitación de éstas, cambian conforme aumenta el nivel de comprensión. En contra de la «intuición realista», conocimiento y realidad son entidades diferentes entre las que no procede hacer correspondencias; como mucho, interpretaciones en términos adaptativos. Los principios de la teoría de Piaget son coherentes con esta visión organicista del conocimiento.

Razones para adoptar el constructivismo organicista u orgánico se han dado con más detalle en otro sitio (Marín, 2003b; Marín, 2005). En el fondo, el constructivismo mecanicista termina manteniendo compromisos epistemológicos con el positivismo (Pozo, 1989; Matthews, 1994) y presenta serias dificultades para explicar la adquisición de nuevos conocimientos y la asignación de significados (Pozo, 2003), que no sólo depende de estructuras conceptuales, sino también de las procedimentales, afectivas $\mathrm{y}$, en general, de todo el organismo biológico del sujeto (Delval, 1997; Castilla del Pino, 2000).

Resulta conveniente destacar en este momento, aunque no se vaya a justificar por no romper con la línea argumental del trabajo, que las posiciones más consensuadas en epistemología de las ciencias también están más cerca del constructivismo orgánico (McComas, Clough y Almazroa, 1998; Fernández et al., 2002; Osborne et al., 2003; Vázquez, Acevedo y Manassero, 2004).

Una vez establecido el marco teórico, el siguiente paso es definir una sistemática de contextos o de aspectos acerca del aprendizaje del alumno a considerar en la formación docente. Éstos se han sintetizado en la tabla 1 donde, además de los trabajos del ámbito de la Didáctica de las Ciencias citados, se han considerado otros de carácter más psicológico (Piaget, 1977; Pozo, 1989; García Madruga, 1990; Delval, 1997; Pozo y Sheuer, 1999; Pérez Echeverría, Pozo y Rodríguez, 2003; Pozo, 2003; Pozo et al., 2006) que han permitido una sistemática de contextos sobre el conocimiento y aprendizaje del alumno más completa. 
Tabla 1

Sistemática de contextos para el cuestionario sobre Vda.

\begin{tabular}{|c|c|c|}
\hline Ind & CONTEXTO O ASPECTO & VISIÓN MÁS ADECUADA \\
\hline 1 & \multicolumn{2}{|c|}{ CORRESPONDENCIA ENTRE OBJETO CONOCIDO (OC) Y OBJETO REAL (OR } \\
\hline 1a & $\begin{array}{l}\text { RELACIÓN ENTRE OBJETO } \\
\text { CONOCIDO (OC) Y REAL (OR) }\end{array}$ & $\begin{array}{c}\text { El acercamiento entre Oc y Or es } \\
\text { sólo adaptativo }\end{array}$ \\
\hline $1 \mathrm{~b}$ & PERCEPCIÓN DE LA REALIDAD & $\begin{array}{l}\text { Sólo es posible acceder a la } \\
\text { realidad construida y ésta es } \\
\text { cambiante }\end{array}$ \\
\hline 2 & \multicolumn{2}{|c|}{ Modelos de ORganización Cognitiva. Certeza del Conocimiento } \\
\hline $2 \mathrm{a}$ & $\begin{array}{l}\text { MODELOS PARA ENTENDER EL } \\
\text { CONOCIMIENTO DEL SUJETO }\end{array}$ & $\begin{array}{c}\text { No REDUCCIONISTAS } \\
\text { Entidad orgánica: el todo es } \\
\text { mayor que la suma de las partes. } \\
\text { Holístico }\end{array}$ \\
\hline $2 b$ & $\begin{array}{l}\text { EL PROBLEMA DE LA CERTEZA } \\
\text { DEL CONOCIMIENTO }\end{array}$ & $\begin{array}{c}\text { LA CERTEZA DE UN } \\
\text { CONOCIMIENTO ES SIEMPRE } \\
\text { RELATIVA }\end{array}$ \\
\hline
\end{tabular}

3 FUENTES DEL CONOCIMIENTO ¿QUÉ SE APRENDE Y DESDE DÓNDE?

\begin{tabular}{|c|c|}
\hline $3 a$ & ORIGEN DEL CONOCIMIENTO \\
\hline $3 b$ & $\begin{array}{c}\text { TIPOS DE INTERACCIONES QUE } \\
\text { FOMENTAN EL DESARROLLO } \\
\text { COGNITIVO } \\
\end{array}$ \\
\hline $3 \mathrm{c}$ & $\begin{array}{l}\text { IMPORTANCIA DE LA ACCIÓN EN } \\
\text { EL DESARROLLO COGNITIVO }\end{array}$ \\
\hline
\end{tabular}

\section{La construcción cognitiva es un proceso genético}

a) interacciones físicas (no necesita la palabra), b) vicarias y c) simbólicas

La acción genera contenidos cognitivos básicos:

procedimentales e implícitos

4 CONTENIDOS COGNITIVOS Y ASIGNACIÓN DE SIGNIFICADOS

¿Todo El Bagaje Cognitivo
SE PUEDE EXPRESAR?

EL PROBLEMA DE LA ASIGNACIÓN DE SIGNIFICADOS

\begin{tabular}{|c|}
\hline $\begin{array}{c}\text { Parte del contenido es } \\
\text { procedimental e implícito }\end{array}$ \\
\hline $\begin{array}{c}\text { Los datos externos siempre } \\
\text { requieren darle sentido, ser } \\
\text { interpretados }\end{array}$ \\
\hline
\end{tabular}

\begin{tabular}{|c|}
\hline 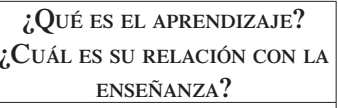 \\
\hline TIPOS DE APRENDIZAJE \\
\hline $\begin{array}{l}\text { GRADOS DE INTEGRACIÓN DE } \\
\text { LO APRENDIDO }\end{array}$ \\
\hline $\begin{array}{c}\text { RELACIONES ENTRE } \\
\text { LO CONSTRUIDO Y LAS } \\
\text { CONDICIONES EXTERNAS }\end{array}$ \\
\hline
\end{tabular}

EL PROCESO DE APRENDIZAJE Y SU RELACIÓN CON LA ENSEÑANZA

\begin{tabular}{|c|}
\hline $\begin{array}{c}\text { Modelos orgánicos ven diferentes } \\
\text { los procesos de enseñar y } \\
\text { aprender }\end{array}$ \\
\hline $\begin{array}{c}\text { Diversidad de aprendizajes y de } \\
\text { integración de lo aprendido }\end{array}$ \\
\hline $\begin{array}{c}\text { Relaciones complejas, pues son } \\
\text { entidades diferentes. Aprender } \\
\text { es difícil }\end{array}$ \\
\hline
\end{tabular}

\section{VISIÓN MENOS ADECUADA}

\begin{tabular}{|c|}
\hline $\begin{array}{c}\text { Existen correspondencias } \\
\text { entre Oc y Or }\end{array}$ \\
\hline $\begin{array}{c}\text { Se puede acceder y cotejar la } \\
\text { realidad externa al sujeto }\end{array}$ \\
\hline
\end{tabular}

realidad externa al sujeto

\begin{tabular}{|c|}
\hline REDUCCIONISTAS \\
Entidad mecánica: el todo es \\
la suma de las partes. \\
Lógicos y simples \\
\hline SE PUEDE COMPROBAR QUE \\
UN CONOCIMIENTO ES MÁs \\
CIERTO QUE OTRO \\
\hline
\end{tabular}

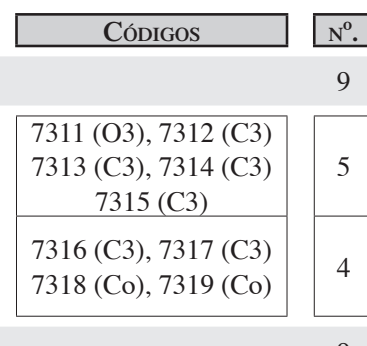

9

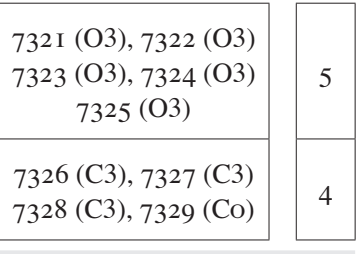

9

Se admite contenido o predisposiciones cognitivas innatas

Predominio de las interacciones simbólicas

El predominio de las interacciones simbólicas resta valor a la acción

\begin{tabular}{|c|}
\hline La mente como procesador \\
simbólico donde la mayor \\
parte o todo es explícito \\
\hline Es posible la apropiación \\
directa: al sujeto llegan \\
significados del exterior \\
\hline
\end{tabular}
significados del exterior

\begin{tabular}{|c|}
\hline $\begin{array}{c}\text { Modelos reduccionistas ven } \\
\text { vínculos lógicos y sencillos } \\
\text { entre enseñar y aprender }\end{array}$ \\
\hline $\begin{array}{c}\text { Reducción a mecánicas } \\
\text { propias de procesadores } \\
\text { simbólicos o verbales }\end{array}$ \\
\hline $\begin{array}{c}\text { Relaciones causales lógicas y } \\
\text { simples EA. Aprender es fácil }\end{array}$ \\
\hline
\end{tabular}

$7331(\mathrm{C} 3), 7332(\mathrm{O} 3)$

$7333(\mathrm{O} 3), 7334$ (O3)

$7335(\mathrm{O} 3), 7336(\mathrm{O} 3)$

$7337(\mathrm{O} 3), 7338(\mathrm{O} 3)$ $7339(\mathrm{O} 3)$

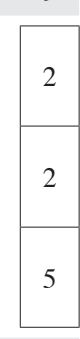

9

\begin{tabular}{|c|c|}
\hline $\begin{array}{c}7411(\mathrm{Co}), 7412(\mathrm{C} 3) \\
7413(\mathrm{O} 3), 7414(\mathrm{Co}) \\
7415(\mathrm{O} 3)\end{array}$ & 5 \\
\hline $\begin{array}{c}7416(\mathrm{O} 3), 7417(\mathrm{O} 3) \\
7418(\mathrm{O} 3), 7419(\mathrm{O} 3)\end{array}$ & \\
\cline { 1 - 1 } & \\
\hline
\end{tabular}

18

\begin{tabular}{|c|c|}
\hline $7421(\mathrm{C} 3), 7422(\mathrm{C} 3)$ & \\
\hline $7423(\mathrm{C} 3), 7424(\mathrm{C} 3)$ & 5 \\
\hline $7425(\mathrm{C} 3)$ & \\
\hline $7426(\mathrm{Co}), 7427(\mathrm{Co})$ & 4 \\
\hline 7428 (C3), 7429 (C3) & 4 \\
\hline 7431 (Co), 7432 (Co) & \\
\hline 7433 (Co), $7434(\mathrm{Co})$ & 6 \\
\hline $7435(\mathrm{Co}), 7436(\mathrm{Co})$ & \\
\hline 7437 (C3), 7438 (C3) & 3 \\
\hline 7439 (C3) & \\
\hline
\end{tabular}

En la tabla 1 se muestra cómo cada contexto o aspecto relevante para ser enseñado viene acompañado de afirmaciones más y menos adecuadas, fundamentadas en el marco teórico desarrollado anteriormente, y de los ítems construidos ex profeso para evaluarlo. Así, por ejemplo, en la primera fila, se sitúa el aspecto «tipos de interacciones que fomentan el desarrollo cognitivo»; al que se le asocia, como visión más adecuada, «las interacciones físicas, vicarias y simbólicas» y como menos adecuada, el «predominio de las interacciones simbólicas». Para evaluar este aspecto concreto, se elaboraron dos ítems (identificados en la base de ítems como 7333 y 7334).

El marco del constructivismo orgánico, usado para desarrollar la tabla 1, es el mismo que el utilizado para la correspondiente tabla del cuestionario elaborado para cono- 
cer la visión de ciencias COMVdC (Marín y Benarroch, 2009). Ahora bien, las diferencias entre la construcción del conocimiento de ciencias y del conocimiento del estudiante (Marín, 2003a) impiden extender más allá el paralelismo entre ambas tablas. Por ejemplo, contextos temáticos tales como las relaciones entre ciencia, tecnología y sociedad, la incorporación de las aportaciones individuales o la regulación de la comunidad científica son más relevantes para entender la construcción del conocimiento de ciencias. Por el contrario, la asignación de significados, el proceso de toma de conciencia o los tipos de aprendizaje son más significativos para comprender la construcción del conocimiento en el estudiante.

Veamos con más detalle cómo se ha elaborado la tabla 1:

- La tabla 1 recoge cinco contextos o aspectos significativos relacionados con el conocimiento del estudiante: a) las relaciones entre objeto conocido y objeto real, b) los modelos sobre organización cognitiva, c) las fuentes del conocimiento, d) la asignación de significados y, finalmente, e) el aprendizaje y su relación con la enseñanza. Como se puede ver, para una mejor estructuración del cuestionario que se pretende elaborar, los contextos se subdividen en temáticas más concretas que se explicitan en la columna 2.

- En total se diseñaron cincuenta ítems, nueve por cada contexto y dieciocho sobre los problemas de aprendizaje y su relación con la enseñanza (ver columna 6), dada la relevancia de este apartado para la enseñanza de las ciencias. De hecho, es el más tratado en la literatura del ámbito de la Didáctica de las Ciencias.

- La columna 3 recoge las afirmaciones más adecuadas para cada contexto, coherentes con la posición del constructivismo orgánico, mientras que la columna 4 contiene las afirmaciones menos adecuadas que, dependiendo de cada contexto, son coherentes con el empirismo, el realismo, el racionalismo, el mecanicismo y, en general, con el reduccionismo. Una argumentación más extensa para justificar la catalogación de las frases de las columnas 3 y 4 en más y menos adecuadas se pueden encontrar en Marín (2003b y 2005).

- La columna 5 contiene los códigos que identifican cada ítem y, entre paréntesis, dos caracteres ( «C $3 » \ll \mathrm{O} 3$ » «Co») que precisan el tipo de estructura usada en el diseño del ítem.

Por la importancia que tiene para el análisis de datos posterior y su interpretación, veamos los detalles y criterios usados para establecer las tres estructuras que han permitido diseñar los ítems del cuestionario que nos ocupa, COMVdA, que, con leves modificaciones, son semejantes y coherentes con las usadas en el cuestionario COMVdC (Marín y Benarroch, 2009):

Ítems con estructura constructivista (C3). Son aquellos ítems que se han diseñado con la siguiente estructura:

a) La primera opción del ítem es una respuesta típicamente constructivista. b) La segunda opción intenta ser más racionalista que la opción primera. En contextos sobre el aprendizaje del alumno se concreta esta opción en la visión interpretati$v a$, la cual supera la acepción de la mente como folio en blanco al percibir el papel activo del conocimiento previo del sujeto para las nuevas adquisiciones, pero se admite que, a pesar de que éstas puedan sesgar o dificultar el aprendizaje, con paciencia y esfuerzo se puede llegar a que el alumno aprenda todo lo que se le quiere enseñar (Pozo y Scheuer, 1999), algo que desde una visión constructivista no se puede asumir.

c) La tercera opción es más empirista que la opción primera.

La opción (a) debe ser coherente con las afirmaciones de la columna 3 de la tabla 1, y las opciones (b) y (c) deben serlo con las afirmaciones de la columna 4 de la misma tabla.

Un ejemplo de ítem «C3» puede verse en el ítem 2 del anexo I, para el que la alternativa a) es la respuesta más racionalista, la b) la empirista, y la c) la más constructivista.

Evidentemente, de cada ítem hay dos versiones. La que usa el investigador en la base de datos, donde siempre la primera opción del ítem es la visión constructivista, tomada como correcta, y la versión que se presenta al alumno, que coincide con la que se muestra en el anexo I, donde la respuesta correcta se «camufla» en cualquier lugar según una clave aleatoria.

Ítems con estructura organicista (O3). Son ítems que abordan problemas ligados a las visiones más o menos complejas que se suelen mantener sobre la construcción del conocimiento del aprendiz de ciencias. Siguen la siguiente estructura:

a) La opción más adecuada al planteamiento formulado en la base del ítem es coherente con el modelo organicista. y, por tanto, coherente con las afirmaciones de la columna 3 de la tabla 1.

b) La segunda y tercera opciones del ítem deben ser respuestas claramente reduccionistas y, por tanto, coherentes con las afirmaciones de la columna 4 de la tabla 1 . Son posiciones coherentes, según el contexto, con el realismo, racionalismo, mecanicismo y, en general, con el reduccionismo.

Un ejemplo de ítem «O3» es el ítem 23 del anexo I.

Ítems con estructura constructivista (Co). No siempre ha sido posible, o quizá no se haya sabido, aplicar las estructuras $\mathrm{C} 3$ u $\mathrm{O} 3$ a determinados ítems, bien porque la temática abordada por la base no lo permitía o porque resultaba difícil en exceso mantener la fórmula para cada opción. En estos casos, se ha optado por una estructura menos rigurosa pero bien definida: la opción más adecuada es coherente con el marco del constructivismo orgánico, mientras que los distractores son incoherentes o menos coherentes. Dichos ítems son de tipo «Co». Ver como ejemplo el ítem 34 del anexo I. 


\section{ADMINISTRACIÓN DEL COMVda EN LA EXPERIENCIA DOCENTE}

El cuestionario que nos ocupa sobre la visión del aprendizaje, COMVdA, forma parte, igual que el que ya se presentó en un trabajo anterior sobre la visión de ciencias, COMVdC (Marín y Benarroch, 2009), de una experiencia de formación con futuros docentes del Curso de Adaptación Pedagógica (asignatura de Física y Química).

El diseño de los primeros ítems de ambos cuestionarios se realizó en 2003, después de sentar los fundamentos que les daban razón de ser (Marín, 2003a; 2003b) y que brevemente se han esbozado en el apartado anterior. Se muestra ahora la secuencia con que se administró el cuestionario COMVdA a partir de 2003.

\section{Primera administración del cuestionario: año 2003}

El objetivo primordial para administrar COMVdA en 2003 fue la depuración de los ítems, lo que implicaba eliminar algunos (se puede ver en la tabla 1 los ítems que se eliminaron, enfatizados en gris en la columna 5) y revisar otros. Así pues, de los cincuenta ítems que contenía el inicial COMVdA, tras su administración a una muestra de 45 futuros profesores de secundaria en enero del 2003, quedaron cuarenta ítems. El cuestionario COMVdA solicita del alumno tres respuestas por cada ítem:

- Primero, la respuesta que se considera correcta.

- Segundo, una valoración de la bondad del ítem. El texto que se entregó a los alumnos fue el siguiente:

- Si has respondido con seguridad, marca (a).

- Si el ítem no parece bien construido, entonces marca:

b) si te parece que entre las opciones no está la respuesta correcta

c) si te parece que hay más de una respuesta correcta.

d) si es que el ítem te parece incoherente por motivos diferentes

- Si no tienes una opinión específica sobre el ítem, entonces marca (e).

En cualquier caso, pero en especial si se ha elegido b), c), o d), se puede realizar un comentario al ítem.

- Tercero, la reacción afectiva del alumnado tras conocer la respuesta «más correcta». Al final del curso, se les informaba sobre qué respuesta hubiera elegido un experto para cada ítem. Las opciones para canalizar su reacción afectiva son: a) He acertado y me siento fenómeno; b) Me parece mejor mi respuesta que la de los expertos (mantengo mi opción); c) Me parece mejor la respuesta de los expertos (cambio mi respuesta); d) No me decido por nada, me siento confuso (confusión o conflicto) y e) El ítem no está bien hecho. No respondo. No me implico. Indiferencia.
Las tres respuestas a cada ítem permitieron el cálculo de dieciséis índices, seis de ellos a partir de la respuesta del alumno al contenido del mismo, cinco relativos a la valoración que hacen de su bondad y cinco sobre la reacción afectiva al escuchar la respuesta correcta. Estos índices fueron utilizados, siguiendo una dinámica muy similar a la descrita en el apartado siguiente, para depurar los cincuenta ítems propuestos en el cuestionario inicial COMVdA y seleccionar los cuarenta ítems que se muestran en el anexo I.

\section{Segunda administración del cuestionario: año 2005}

Si en el 2003 el objetivo era depurar y validar los ítems, en 2005 las metas se amplían; ahora se trata de usar la nueva herramienta en la experiencia docente para animar el aprendizaje y evaluar lo aprendido. Por esta razón, el cuestionario COMVda se pasa al inicio del curso como pretest y al final como postest. Entre ellos, existe una fase donde el cuestionario se usa en clase como un recurso didáctico en un contexto de enseñanza más amplio. La secuencia seguida fue la siguiente:

- Individualmente, se interactúa por primera vez con el cuestionario usado como pretest (Anexo I). Cada ítem se responde de dos modos: además de la elección de la respuesta correcta, se pide una valoración de la percepción de la bondad de cada ítem (tal y como se ha explicado para 2003).

- En pequeños grupos, se debaten las valoraciones individuales a cada ítem a fin de buscar consensos que son registrados y presentados al profesor en un informe. Con esta actividad, se procura también el debate grupal sobre las cuestiones problemáticas planteadas y sus posibles soluciones; en definitiva, se pretende una primera interiorización y familiarización con el tema.

- Se exponen a toda la clase los contenidos teóricos sobre «conocimiento y aprendizaje del alumno». Se pretende, entre la exposición y los debates, llevar a los alumnos hacia posiciones cercanas al constructivismo orgánico (Marín, 2003b).

- De nuevo se interactúa individualmente con el cuestionario ahora usado como postest. También se piden dos respuestas por cada ítem: la primera, al elegir la que se considera respuesta correcta y, la segunda, mostrando su reacción afectiva una vez que el profesor indica qué opción elige el experto (tal y como se ha explicado para 2003).

El que ahora haya pretest y postest hace aumentar de dieciséis a veintidós los índices que permiten analizar la bondad de cada ítem. En el trabajo que antecede a éste se dio una descripción detallada de cada índice (Marín y Benarroch, 2009), por lo que aquí nos limitamos a una breve alusión:

\section{A. Índices obtenidos de COMVdA aplicado como pretest}

- Asociados a la elección de la respuesta correcta, se obtienen las frecuencias con que se han elegido cada una de las opciones. Son los índices Ia, Ib e Ic. La frecuencia de los que no han respondido al ítem dio lugar al índice IO. 
Asimismo, se calcularon dos índices de discriminación que miden el comportamiento del ítem frente al resultado total: ID e IE. Esto supone un total de seis índices.

- Asociados a la valoración de la calidad de cada ítem, se construyeron cinco índices, a saber, Va (recoge el porcentaje de alumnos que piensa que el ítem está bien construido), $\mathrm{Vb}$ (porcentaje de alumnos que piensan que al ítem le faltan opciones), Vc (porcentaje que piensa que el ítem tiene más de una respuesta correcta), $\mathrm{Vd}$ (porcentaje que opina que el ítem no está bien construido por otros motivos) y Ve (porcentaje que no tiene una opinión específica sobre el ítem).

\section{B. Índices obtenidos de COMVdA aplicado como postest}

- Los mismos índices que se han calculado en el pretest, asociados a la elección de la respuesta correcta. Esto supone un total de seis índices Ia, Ib, Ic, I0, ID e IE pero ahora calculados con los resultados del postest.

- Índices asociados a la reacción afectiva al conocer la respuesta dada por los expertos. Así, se distinguen Sa («estupendo, acerté»), Sb («sigo manteniendo mi opción»), $\mathrm{Sc}$ («creo que es mejor la respuesta de los expertos»), Sd («esto lo veo confuso») y Se («no sé qué decir»).

\section{VALORACIÓN DE LOS ÍTEMS DEL CUES- TIONARIO COMVda}

Una vez administrado el cuestionario COMVdA (anexo I) como pretest y como postest en el 2005, y calculados los veintidós índices que se explican en el apartado anterior, se trató de encontrar la forma de valorar los ítems a partir de esos índices incompatibles entre sí. El problema reside en que no son compatibles entre sí, ya que unos reflejan decisiones cognitivas, otros estimaciones y otros tendencias afectivas; por tanto, ¿cómo se puede objetivizar el proceso para que sea aceptado interpersonalmente?

Tras analizar varias opciones, la mejor solución encontrada supuso dar los siguientes pasos:

- Primer paso: se agrupan los índices por factores, cada uno representando una cualidad del ítem. La suma de todos daría un valor global sobre la bondad y eficacia del ítem. Los factores son los siguientes:

Factor 1: Capacidad del ítem para conseguir una distribución equitativa del porcentaje de elecciones entre sus opciones. Se usan para calcular este factor los índices Ia, Ib e Ic del pretest.

Factor 2: Capacidad de discriminación del ítem. Se usan Ia, ID e IE del pretest.

Factor 3: Valoración del ítem por el encuestado. Se usa para calcular dicho índice $\mathrm{Va}, \mathrm{Vb}, \mathrm{Vc}, \mathrm{Vd}$ e $\mathrm{I}$.

Factor 4: Cualidad para distribución equitativa del porcentaje de elecciones entre las opciones. Se usan los índices Ia, Ib e Ic del postest.
Factor 5: Capacidad de discriminación del ítem. Se usan Ia, ID e IE del postest.

Factor 6: Grado de implicación del alumno al conocer la opción del experto. Se usan los índices Sc, Sd y Se.

Se ha procurado diseñar los factores de forma que tengan un peso específico similar en la calidad del ítem.

- Segundo paso: se estima el valor de cada factor a partir de los índices que representa. Esta estimación es realizada por seis grupos independientes constituidos por dos expertos en el tema y cuatro grupos de estudiantesprofesores. Para ello, se aceptan unos límites para esos índices, de forma que, de modo combinado, el factor adquiera un valor que se acerque a la valoración cualitativa previa realizada a cada ítem (ver procedimiento en Marín y Benarroch, 2009).

- Tercer paso: se calcula el nivel de calidad del índice a partir de la suma de los valores adquiridos por los respectivos factores. Después se realiza una reducción de escala a fin de disminuir la probabilidad de clasificaciones incorrectas. Los ítems que hayan logrado una puntuación en el rango [4,6 - 6] son los ítems de más calidad y se le asigna el nivel 1 . Los del rango [2,6 - 4,5] tienen nivel $2 \mathrm{y}$, finalmente, con [0 - 2,5] se les asigna el nivel 3, que es el más bajo.

La tabla 2 muestra los resultados de la valoración de los ítems. Las columnas indican, respectivamente: orden del ítem en el cuestionario, código del ítem (el mismo de la tabla 1), tipo de ítem, las valoraciones de los seis grupos de jueces, la media aritmética de estas valoraciones y, finalmente, la calidad del ítem según escala explicada anteriormente. Obsérvese que sólo hay tres ítems de nivel 3 , y por tanto, de baja calidad.

La tabla 3 es una tabla aclaratoria con ejemplos sobre el proceso seguido. En las columnas 1, 2 y 3 se muestran los códigos de los ítems clasificados por niveles y según su tipo. Como se puede comprobar, los resultados de la valoración de los ítems son:

- Nivel 1: 15 ítems C3, 9 ítems O3 y 4 ítems Co

- Nivel 2: 5 ítems O3 y 4 ítems Co

- Nivel 3: 1 ítem C3, 1 ítem O3 y 1 ítem Co

En la columna 4 de la misma tabla 3, se muestra un ítem representativo de cada tipo y nivel, así como un comentario sobre el proceso seguido de su valoración a partir de los índices.

En la tabla 4 se muestran las correlaciones entre ítems tras la administración del cuestionario en 2003 y 2005 y entre pretest y postest del 2005. Las correlaciones altas serían manifestaciones de un comportamiento estable o fiable del cuestionario COMVdA ante su administración a diferentes muestras, y las correlaciones bajas serían indicativas de un comportamiento azaroso que podría estar asociado a una deficiente construcción de los ítems cuyas opciones no las 
vería el entrevistado como buenas propuestas de decisión cognitiva. Si predominaran las correlaciones bajas (que, como veremos, no es el caso), el esfuerzo de clasificar los ítems por niveles de calidad no tendría mucho sentido al no ser estable su comportamiento.

Salvo pocas excepciones, que serán comentadas a continuación, la tabla 4 muestra buenas correlaciones significativas al $95 \%$ de confianza. Veamos los detalles:

- Las correlaciones más importantes son las asociadas a los índices Ia, Ib, Ic e I0 y vemos que son buenas o muy buenas. Las únicas excepciones se encuentran en las bajas correlaciones de I0 y de ID entre 2003 y 2005, lo que se debe a la revisión de ítems que media entre estos años, revisión que puede haber originado una mayor aceptación de las nuevas propuestas de opciones. Este problema se soluciona en el 2005, donde las correlaciones entre el pretest y el postest en todos los índices son excepcionalmente altas, poniendo de manifiesto que el cuestionario COMVdA, tal y como se muestra en el anexo I, tiene un comportamiento muy fiable.

- Las correlaciones desiguales asociadas a los índices V (percepción de la bondad de la construcción del ítem) y S (reacción afectiva al conocer la respuesta correcta), se deben a que estos índices recogen percepciones subjetivas y afectivas del entrevistado, mientras que Ia, Ib e Ic representan esfuerzos más bien de tipo cognitivo. Estos últimos índices, al ser supuestamente más racionales, son más significativos para valorar la estabilidad del comportamiento de COMVdA.

Tabla 2

Valoración de ítems sobre Vda.

\begin{tabular}{|c|c|c|c|c|c|c|c|c|c|}
\hline $\begin{array}{c}\text { ORDEN } \\
\text { EN PRUEBA } \\
\end{array}$ & $\begin{array}{c}\text { CÓDIGO } \\
\text { ÍTEM }\end{array}$ & $\begin{array}{c}\text { TIPO DE } \\
\text { ÍTEM }\end{array}$ & $\begin{array}{c}\text { GRUPO } \\
N^{0} .1 \\
\end{array}$ & $\begin{array}{c}\text { GRUPO } \\
\mathrm{N}^{\mathrm{o}} .2 \\
\end{array}$ & $\begin{array}{c}\text { GRUPO } \\
\mathbf{N}^{\circ} .3 \\
\end{array}$ & $\begin{array}{c}\text { GRUPO } \\
N^{0} .4 \\
\end{array}$ & $\begin{array}{c}\text { GRUPO } \\
\mathbf{N}^{0} .5 \\
\end{array}$ & $\begin{array}{c}\text { MEDIA } \\
\text { ARITMÉTICA } \\
\end{array}$ & $\begin{array}{c}\text { NIVEL } \\
\text { DE CALIDAD } \\
\end{array}$ \\
\hline 1 & 7312 & C3 & 6 & 6 & 6 & 6 & 6 & 6 & 1 \\
\hline 2 & 7313 & $\mathrm{C} 3$ & 6 & 6 & 6 & 6 & 6 & 6 & 1 \\
\hline 3 & 7314 & C3 & 5,5 & 5,5 & 5,5 & 6 & 5,5 & 5,6 & 1 \\
\hline 4 & 7315 & $\mathrm{C} 3$ & 5 & 5 & 5 & 6 & 6 & 5,4 & 1 \\
\hline 5 & 7316 & C3 & 4,5 & 4,5 & 4,5 & 4,5 & 5 & 4,6 & 1 \\
\hline 6 & 7317 & C3 & 6 & 6 & 5,5 & 6 & 6 & 5,9 & 1 \\
\hline 7 & 7318 & Co & 6 & 6 & 6 & 6 & 6 & 6 & 1 \\
\hline 8 & 7319 & Co & 5,5 & 5,5 & 5,5 & 6 & 5,5 & 5,6 & 1 \\
\hline 9 & 7321 & $\mathrm{O} 3$ & 5 & 5 & 4,5 & 5,5 & 5 & 5 & 1 \\
\hline 10 & 7322 & $\mathrm{O} 3$ & 5,5 & 5,5 & 6 & 6 & 5,5 & 5,7 & 1 \\
\hline 11 & 7324 & $\mathrm{O} 3$ & 6 & 6 & 6 & 5,5 & 6 & 5,9 & 1 \\
\hline 12 & 7325 & $\mathrm{O} 3$ & 5 & 6 & 4,5 & 5 & 5 & 5,1 & 1 \\
\hline 13 & 7326 & $\mathrm{C} 3$ & 6 & 6 & 6 & 5,5 & 6 & 5,9 & 1 \\
\hline 14 & 7327 & C3 & 6 & 6 & 6 & 5,5 & 6 & 5,9 & 1 \\
\hline 15 & 7331 & $\mathrm{C} 3$ & 5 & 5 & 4,5 & 6 & 5 & 5,1 & 1 \\
\hline 16 & 7333 & $\mathrm{O} 3$ & 2,5 & 2,5 & 3,5 & 2,5 & 3 & 2,8 & 2 \\
\hline 17 & 7334 & $\mathrm{O} 3$ & 3 & 3 & 3 & 3 & 3 & 3 & 2 \\
\hline 18 & 7336 & $\mathrm{O} 3$ & 4,5 & 4,5 & 4 & 4,5 & 4,5 & 4,4 & 2 \\
\hline 19 & 7337 & $\mathrm{O} 3$ & 4,5 & 4,5 & 4 & 5,5 & 5 & 4,7 & 1 \\
\hline 20 & 7338 & $\mathrm{O} 3$ & 2 & 2 & 3 & 2 & 2 & 2,2 & 3 \\
\hline 21 & 7339 & $\mathrm{O} 3$ & 5,5 & 5,5 & 5,5 & 5,5 & 5 & 5,4 & 1 \\
\hline 22 & 7411 & $\mathrm{Co}$ & 4 & 4 & 3,5 & 4 & 4 & 3,9 & 2 \\
\hline 23 & 7413 & $\mathrm{O} 3$ & 5,5 & 5,5 & 5,5 & 5,5 & 5,5 & 5,5 & 1 \\
\hline 24 & 7415 & $\mathrm{O} 3$ & 4,5 & 4,5 & 4,5 & 4,5 & 4,5 & 4,5 & 2 \\
\hline 25 & 7416 & $\mathrm{O} 3$ & 5 & 5 & 5 & 5 & 5 & 5 & 1 \\
\hline 26 & 7417 & $\mathrm{O} 3$ & 3 & 3 & 3,5 & 3,5 & 3,5 & 3,3 & 2 \\
\hline 27 & 7419 & $\mathrm{O} 3$ & 5 & 5 & 5 & 5 & 5 & 5 & 1 \\
\hline 28 & 7421 & C3 & 5 & 5 & 5 & 4,5 & 5 & 4,9 & 1 \\
\hline 29 & 7423 & $\mathrm{C} 3$ & 2 & 2 & 3,5 & 2,5 & 2,5 & 2,5 & 3 \\
\hline 30 & 7424 & $\mathrm{C} 3$ & 5 & 5 & 5,5 & 5 & 5,5 & 5,2 & 1 \\
\hline 31 & 7426 & Co & 4,5 & 4,5 & 5 & 4,5 & 5 & 4,7 & 1 \\
\hline 32 & 7427 & $\mathrm{Co}$ & 2,5 & 2,5 & 3,5 & 3,5 & 3,5 & 3,1 & 2 \\
\hline 33 & 7428 & $\mathrm{C} 3$ & 5,5 & 5,5 & 5,5 & 6 & 5,5 & 5,6 & 1 \\
\hline 34 & 7431 & Co & 4 & 4 & 4 & 3,5 & 4 & 3,9 & 2 \\
\hline 35 & 7432 & Co & 4 & 4 & 4 & 4,5 & 4 & 4,1 & 2 \\
\hline 36 & 7435 & Co & 2 & 2 & 3 & 2 & 2 & 2,2 & 3 \\
\hline 37 & 7436 & Co & 5 & 5 & 5 & 4,5 & 5 & 4,9 & 1 \\
\hline 38 & 7437 & $\mathrm{C} 3$ & 5,5 & 5,5 & 5,5 & 5,5 & 5,5 & 5,5 & 1 \\
\hline 39 & 7438 & $\mathrm{C} 3$ & 4,5 & 4,5 & 4,5 & 5 & 4,5 & 4,6 & 1 \\
\hline 40 & 7439 & $\mathrm{C} 3$ & 5,5 & 5,5 & 5 & 5,5 & 5 & 5,3 & 1 \\
\hline
\end{tabular}


Tabla 3

Clasificación y ejemplos de ítems de la prueba COMVda.

\begin{tabular}{|c|c|c|c|}
\hline NIVEL & TIPO & CÓDIGOS & EJEMPLOS \\
\hline \multirow{3}{*}{1} & $\begin{array}{c}C 3 \\
\text { (15) }\end{array}$ & $\begin{array}{l}n^{o} .01: 7312 \\
n^{o} .02: 7313 \\
n^{o} .03: 7314 \\
n^{o} .04: 7315 \\
n^{o} .05: 7316 \\
n^{o} .06: 7317 \\
n^{o} .13: 7326 \\
n^{o} .14: 7327 \\
n^{o} .15: 7331 \\
n^{o} .28: 7421 \\
n^{o} .30: 7424 \\
n^{o} .33: 7428 \\
n^{o} .38: 7437 \\
n^{o} .39: 7438 \\
n^{o} .40: 7439\end{array}$ & $\begin{array}{l}\text { Ítem } \mathrm{n}^{\circ} \text {. 03: } 7314 \text {. La imagen que el sujeto tiene de una silla es como: } \\
\text { a) una idea útil pero no se sabe si es o no imagen de la silla } \\
\text { b) una fotografía de la silla más o menos distorsionada } \\
\text { c) una copia parcial de la silla cada vez más completa } \\
\text { Comentario: Un ítem excelente. Los resultados en } 2003 \text { son un tanto discretos y se manifiesta como un ítem } \\
\text { relativamente bueno: (Ia:59 b:14 c:03 0:22 D:25 E:25 / Va:25 Vb:07 Vc:14 Vd:07 V0:44 B / Sa:62 b:14 c:11 } \\
\text { d:07 e:03). } \\
\text { Tras una ligera revisión, los índices para } 2005 \text { manifiestan una notable mejoría: (Ia:20 b:16 c:52 0:09 D:33 } \\
\text { E:71 / Va:67 b:05 c:14 d:04 e:10 / Ia:26 b:14 c:56 0:04 D:35 E:45 / Sa:24 b:36 c:33 d:05 e:05). Aplicando } \\
\text { los criterios establecidos para cada factor, se observa que los porcentajes de elección se reparten entre a, b y } \\
\text { c. La discriminación es muy buena. Es bastante bien valorado por los alumnos y manifiesta un buena actitud } \\
\text { para incitar al aprendizaje. }\end{array}$ \\
\hline & $\begin{array}{l}O 3 \\
(9)\end{array}$ & $\begin{array}{l}n^{o} .09: 7321 \\
n^{o} .10: 7322 \\
n^{o} .11: 7324 \\
n^{o} .12: 7325 \\
n^{o} .19: 7337 \\
n^{o} .21: 7339 \\
n^{o} .23: 7413 \\
n^{o} .25: 7416 \\
n^{o} .27: 7419\end{array}$ & $\begin{array}{l}\text { Ítem n }{ }^{\circ} 10: 7322 \text {. La relación entre las partes que integran el conocimiento es tal que su suma: } \\
\text { a) Es menos que el todo, como los órganos de un ser vivo } \\
\text { b) Es el todo, como el encaje de las piezas de un reloj } \\
\text { c) Es el todo, como el encaje de las piezas de un puzle } \\
\text { Comentario: El ítem, a pesar de su dificultad, se elige en } 2003 \text { por su buena capacidad de discriminación } \\
\text { (Ia:11 b:48 c:25 0:14 D:25 E:99 / Va:33 Vb:03 Vc:14 Vd:07 V0:40 / Sa:11 b:48 c:11 d:14 e:03). } \\
\text { Tras la revisión de las opciones a y b, el ítem mejora sus índices cuando se pasa en } 2005 \text { (Ia:38 b:20 c:27 0:12 } \\
D: 33 \text { E:45 / Va:63 b:01 c:08 d:11 e:17 / Ia:45 b:26 c:22 0:05 D:60 E:69 / Sa:49 b:09 c:29 d:12 e:02). Obsérvese } \\
\text { que los porcentajes de elección se distribuyen con bastante equidad entre a, b y c. La discriminación es } \\
\text { bastante buena sobre todo tras la experiencia de aprendizaje. Los alumnos lo valoran muy bien y, observando } \\
\text { «Sc+Sd» y el bajo «Se» podemos decir que es un ítem bastante motivador para el aprendizaje del alumno. }\end{array}$ \\
\hline & $\begin{array}{l}\text { Co } \\
\text { (4) }\end{array}$ & $\begin{array}{l}n^{o} .07: 7318 \\
n^{o} .08: 7319 \\
n^{o} .31: 7426 \\
n^{o} .37: 7436\end{array}$ & $\begin{array}{l}\text { Ítem no08: } 7319 \text { : Un cambio sustancial en mi conocimiento de la realidad: } \\
\text { a) cambiará mi modo de afrontar la realidad } \\
\text { b) hará que conozca de un modo más preciso la realidad } \\
\text { c) me permitirá conocer más la verdad de la realidad } \\
\text { Comentario: El ítem fue elegido en } 2003 \text { por sus muy buenos resultados (Ia:25 b:25 c:04 0:44 D:36 E:53 } \\
\text { Va:20 Vb:09 Vc:03 Vd:02 V0:67 / Sa:30 b:13 c:05 d:31 e:00). } \\
\text { Los mismos buenos resultados los mantiene en } 2005 \text { (Ia:61 b:20 c:16 0:01 D:53 E:44 / Va:69 b:01 c:22 d:02 e:06 } \\
\text { Ia:62 b:30 c:07 0:00 D:66 E:62 / Sa:60 b:15 c:21 d:02 e:02). Pone de manifiesto también una notable fiabilidad. }\end{array}$ \\
\hline \multirow{2}{*}{2} & $\begin{array}{l}O 3 \\
(5)\end{array}$ & $\begin{array}{l}n^{o} .16: 7333 \\
n^{o} .17: 7334 \\
n^{o} .18: 7336 \\
n^{o} .24: 7415 \\
n^{o} .26: 7417\end{array}$ & $\begin{array}{l}\text { Ítem } n^{\circ} 18: 7336: \text { Principalmente, además de la ayuda de los mayores, ¿qué es lo que más influye para } \\
\text { aprender a andar? } \\
\text { a) evitar golpes de las caídas } \\
\text { b) instinto heredado para andar } \\
\text { c) impulso de imitar a los mayores } \\
\text { Comentario: El ítem se muestra fiable y discrimina bien a pesar de ser un ítem difícil. En } 2003 \text { (Ia:29 b:07 } \\
\text { c:33 0:29 D:12 E:99 / Va:25 Vb:11 Vc:22 Vd:11 V0:29 / Sa:37 b:18 c:14 d:07 e:18) } \\
\text { En } 2005 \text { (Ia:12 b:41 c:34 0:10 D:20 E:99 / Va:60 b:05 c:24 d:02 e:10 / Ia:11 b:45 c:41 0:01 D:26 E:99 / } \\
\text { Sa:11 b:54 c:23 d:08 e:04) }\end{array}$ \\
\hline & $\begin{array}{l}\text { Co } \\
\text { (4) }\end{array}$ & $\begin{array}{l}n^{o} .22: 7411 \\
n^{o} .32: 7427 \\
n^{o} .34: 7431 \\
n^{o} .35: 7432\end{array}$ & $\begin{array}{l}\text { Ítem n } \mathrm{n}^{\circ 2} \text { : 7411: En general, ¿hay diferencias entre lo que se adquiere por vivencia personal y por información? } \\
\text { a) Sí, lo primero da más habilidades; lo segundo, da más ideas } \\
\text { b) Sí, el grado de valor y utilidad que tienen para el sujeto } \\
\text { c) No, todo se va añadiendo en un entramado de ideas } \\
\text { Comentario: Aunque el alumno no tiene buena percepción del ítem, reparte y discrimina muy bien en } 2003 \\
\text { (Ia:25 b:33 c:14 0:25 D:37 E:60 / Va:25 Vb:22 Vc:22 Vd:00 V0:29 / Sa:33 b:22 c:22 d:11 e:07). } \\
\text { Revisado para } 2005 \text { cambia sensiblemente su comportamiento a peor (Ia:49 b:30 c:12 0:07 D:13 E:11 / } \\
\text { Va:67 b:03 c:11 d:00 e:19 / Ia:58 b:28 c:13 0:00 D:00 E:00 / Sa:56 b:15 c:27 d:00 e:02) }\end{array}$ \\
\hline \multirow[b]{3}{*}{3} & $\begin{array}{l}\text { C3 } \\
\text { (1) }\end{array}$ & $n^{o} .29: 7423$ & \multirow{3}{*}{$\begin{array}{l}\text { Ítem n²9: 7423: Sobre cualquier objeto, ¿siempre se puede aprender algo más? } \\
\text { a) Sí, nuevas experiencias pueden aportar más información } \\
\text { b) No, sólo sería cierto para objetos complejos } \\
\text { c) Depende, hay objetos de los que se posee una imagen completa } \\
\text { Comentario: En } 2003 \text { el ítem fue elegido por su discriminación más que por otros factores (Ia:74 b:00 c:03 } \\
0: 22 \text { D:37 E:27 / Va:37 Vb:14 Vc:00 Vd:03 V0:44 / Sa:74 b:00 c:11 d:00 e:11). } \\
\text { Tras la revisión, en } 2005 \text {, la opción (a) se sigue mostrando muy atractiva y es baja la habilidad para incitar } \\
\text { el aprendizaje (Ia:85 b:03 c:07 0:03 D:46 E:30 / Va:78 b:00 c:06 d:04 e:13 / Ia:94 b:00 c:03 0:01 D:13 } \\
\text { E:07 / Sa:90 b:01 c:06 d:00 e:02) }\end{array}$} \\
\hline & $\begin{array}{l}O 3 \\
(1) \\
\end{array}$ & $n^{o} .20: 7338$ & \\
\hline & $\begin{array}{l}\text { Co } \\
\text { (1) }\end{array}$ & $n^{o} .36: 7435$ & \\
\hline
\end{tabular}


Tabla 4

Correlaciones de índices de COMVda.

\begin{tabular}{|c|c|c|c|c|}
\hline \multicolumn{5}{|c|}{ CORRELACIONES ENTRE 2003 Y 2005} \\
\hline $\mathrm{Ia} 03-\mathrm{Ia} 05$ & $\mathrm{Ib} 03-\mathrm{Ib} 05$ & Ic03-Ic05 & Io03-Io05 & ID03-ID05 \\
\hline $0,580^{* *}$ & $0,676^{* *}$ & $0,398 * *$ & $-0,134$ & 0,076 \\
\hline $\mathrm{Va} 03-\mathrm{Va} 05$ & Vb03-Ib05 & $\mathrm{Vc03-Vc05}$ & Vd03-Vd05 & $\mathrm{Ve} 03-\mathrm{Ve} 05$ \\
\hline 0,14 & 0,233 & $0,658 * *$ & $0,387 *$ & 0,074 \\
\hline $\mathrm{Sa03-Sa05}$ & $\mathrm{Sb03-Sb05}$ & $\mathrm{Sc} 03-\mathrm{Sc} 05$ & $\mathrm{Sd} 03-\mathrm{Sd} 05$ & $\mathrm{Se} 03-\mathrm{Se} 05$ \\
\hline $0,562 * *$ & $0,334 *$ & $0,388^{*}$ & 0,117 & 0,188 \\
\hline \multicolumn{5}{|c|}{ CORRELACIONES ENTRE PRETEST Y POSTEST DEL 2005} \\
\hline Ia-Ia’ & Ib-Ib' & Ic-Ic' & Io-Io' & ID-ID' \\
\hline $0,933^{* *}$ & $0,924 * *$ & $0,901 * *$ & $0,683^{* *}$ & $0,682^{* *}$ \\
\hline
\end{tabular}

\section{RESULTADOS DEL APRENDIZAJE DE LOS ALUMNOS EN LA EXPERIENCIA DOCENTE}

En este apartado se desarrollará el sentido más práctico del cuestionario COMVdA, puesto que permite analizar el progreso del aprendizaje durante la experiencia docente así como mostrar detalles sobre el progreso de los estudiantes en función de sus actitudes epistemológicas. Concretamente:

a) Evaluación de los aprendizajes realizados durante la experiencia docente en 2005.

Como se ha explicado con anterioridad, para cumplir con este objetivo, el cuestionario fue administrado como pretest y postest (una hora para su administración en cada caso) de un proceso de enseñanza sobre el conocimiento y el aprendizaje del alumno de 10 horas de duración.

Los cálculos de las puntuaciones de los alumnos toman como punto de partida la tabla 5. En ella, se muestra, a mano izquierda, los datos del pretest obtenidos por cada sujeto y, en la derecha, los del postest. Para cada uno de ellos, se recoge la matriz de casos y variables, porcentaje de aciertos, porcentaje de errores, porcentaje de opciones $\mathrm{b}$, porcentaje de opciones c, porcentaje de respuestas en blanco, nota obtenida por el alumno, y la nota de unos parciales P1 y P2, cuyo significado se verá después. La nota total se obtiene del siguiente modo: aciertos - (errores/número de distractores). Por tanto:

$$
\text { Nota }=I a-\frac{E r}{n^{o} . \text { distractores }}=I a-\frac{E r}{2}
$$

Si se comparan las notas obtenidas por los alumnos en el postest frente al pretest, mediante la prueba $\mathrm{T}$ para muestras relacionadas, con el SPSS 15.0, se obtiene una diferencia de medias de 10,83 que es significativa al 99\% de confianza. Para ponderar adecuadamente este valor, merece la pena compararlo con la media obtenida en el pretest -21,75-, constatando un avance en los conocimientos del $50 \%$, lo que supone un incremento de casi la mitad.

Se puede concluir que la propuesta de enseñanza es, considerando el tiempo disponible, suficientemente efectiva.

b) Evaluación del progreso constructivista y organicista de los alumnos. Una aplicación importante del cuestionario COMVdA es que posibilita evaluar los progresos que, a nivel global, ha experimentado el grupo de alumnos en sus visiones constructivistas y organicistas. Para ello, es necesario calcular unas notas parciales a partir de los ítems construidos según las estructuras-tipo. En concreto, se deben considerar dos grupos de ítems:

- Los de tipo C3 y calidad 1 o 2 (en total 15 ítems). La puntuación de cada alumno obtenida a partir de este grupo de ítems ha sido denominada Nota del Parcial 1.

- Los de tipo O3 y calidad 1 o 2 (en total 14 ítems). La puntuación de cada alumno obtenida a partir de este grupo de ítems ha sido denominada Nota del Parcial 2.

En la tabla 5 figuran estas notas parciales, tanto en el pretest como en el postest. El análisis de los progresos constructivistas se realiza usando tan sólo las notas del parcial 1. Si se comparan éstas (columnas P1 del pretest y del postest de la tabla 5) mediante la prueba $\mathrm{T}$ para muestras relacionadas, con el SPSS 15.0, se obtienen una diferencia de medias de 5,26 que sólo es significativa al $90 \%$ de confianza. 
Tabla 5

Cálculos de notas para prestest y postest de COMVda

Resultados del Pretest de COMVda

\begin{tabular}{|ccccccc|c|}
\hline S & Matriz de casos y variables & Ia & Er & Ib & Ic & I0 \\
\hline 02 & caac0aaa0a0aaaabcb0aabaaaaaaaabbcbaaabab & 60 & 30 & 20 & 10 & 10
\end{tabular}

03 cbbcbc0aca0ccccaaaaaabaacabcab0aca0aa0cb $40 \quad 47 \quad 17 \quad 30 \quad 12$

04 ccb0ccaa0acbbccc0b0c00aaba00bbbab0aaacab $27 \quad 50 \quad 252522$

05 bcccacbacbaaaaccaccaabbaaaaaabbaccaaaacb $\begin{array}{llllll}50 & 50 & 20 & 30 & 0\end{array}$

06 abbcacacababaccabaababbaccacabaacaabcccb $42 \quad 57 \quad 27 \quad 30 \quad 0$

07 ab000abac000a0ca0b0c0cac0aa00b0a0a0000b0 $25 \quad 25 \quad 12 \quad 12 \quad 50$

$\begin{array}{lllllll}08 & \text { caabbaaaaabaccaaacaaabcaabaaaaaaaaacaa } & 72 & 27 & 12 & 15 & 0\end{array}$

$\begin{array}{lllllll}09 & \text { caabbbbbbcbcbababbbcabcabaccabbbcaacacbc } & 27 & 72 & 45 & 27 & 0\end{array}$

10 ab0cbb0abaabaa0accaca00cca00aaa0cc0baaca $40 \quad 37 \quad 15 \quad 22 \quad 22$

11 cccbcc0a0aabbcca00aa0abbbaaacbbac0a0bccb $30 \quad 52 \quad 25 \quad 27 \quad 17$

13 cabcbbabbcbcbabaabbaac0aaaaaabbac0a0aaac $45 \quad 47 \quad 30 \quad 17 \quad 7$

14 abbbccbaaaa0ccbaacaaaba0cbaaaaabcaaacc0b $47 \quad 45 \quad 22 \quad 22 \quad 7$

15 cccbbcbb0aabbbcbabaaaacbaaabaa0acaaabbba $42 \quad 52 \quad 35 \quad 17 \quad 5$

16 ccabbababaabaacaabcaaacbaabaabbaaaabac0b $\begin{array}{llllll}52 & 45 & 30 & 15 & 2\end{array}$

17 c0bbacbc0ccbcbcaabacaaaaabababbac0cbb0aa $35 \quad 55 \quad 30 \quad 25 \quad 10$

$18 \quad$ ccacacaaabbccacaababaaaaaaaacaaaaacccaa $62 \quad 37 \quad 10 \quad 27 \quad 0$

19 aaabbc0aaaabaaaaaba0aabaab0aa0baaaaaabac $\begin{array}{llllll}65 & 25 & 20 & 5 & 10\end{array}$

20 c0cc00bb0ccbacc0a0acaa0bacb0abba0aa0bcab $27 \quad 47 \quad 22 \quad 2525$

21 c0bbacbaabbbccbaabccaaaabb0ca00acaabb0ba $\begin{array}{llllll}35 & 52 & 32 & 20 & 12\end{array}$

22 cabbbcaabcbbbccaaacaaabcaaa0abbacbabcc0a $40 \quad 55 \quad 30 \quad 25 \quad 5$

23 ca0bac0b0cbca00aabaaaaaa0000a0baaa0aaaba $47 \quad 25 \quad 15 \quad 10 \quad 27$

24 c0ab0acab0a0a0caa0a0ab00aa00abb0c00b0abc $32 \quad 30 \quad 17 \quad 12 \quad 37$

$\begin{array}{lllllll}25 & \text { cccbccabbc0babb0acbbabbaba0bccaaaaaac00a } & 32 & 55 & 30 & 25 & 12\end{array}$

$\begin{array}{lllllll}26 & \text { cabcbbaa0babaacaacaaabaaacbaaabaccaabbaa } & 55 & 42 & 25 & 17 & 2\end{array}$

27 cbcbbcaa0a0ca0a0acacab0cabaaac0aababbbcc $37 \quad 47 \quad 22 \quad 25 \quad 15$

28 caacba0ac0cbaacaabaacaaabaaaaaaabcaaa0ac $60 \begin{array}{lllll}60 & 32 & 12 & 20 & 7\end{array}$

$\begin{array}{lllllll}30 & \text { ababccac0ccbabcabcacaabaaabacbbabaabccca } & 40 & 57 & 27 & 30 & 2\end{array}$

$\begin{array}{lllllll}31 & \text { aacbbcbcacba0aaaabccacbbacba0baacbccbcba } & 35 & 60 & 30 & 30 & 5\end{array}$

$\begin{array}{lllllll}32 & \text { baccccbabccaaacacbacabacacbaabbacaaacaaa } & 47 & 52 & 20 & 32 & 0\end{array}$

33 ccacbcaabbabaccaacaaaba0aa0aa0b0aaa0aaaa $\begin{array}{llllll}55 & 32 & 15 & 17 & 12\end{array}$

34 abbbbcca0ab0cbbaac0c0cabaa0bab00aaaaa0cb $35 \quad 45 \quad 27 \quad 17 \quad 20$

35 cbbbbbbbcccccccaacaaabaaaaacacaacbaabcba $40 \quad 60 \begin{array}{lllll}47 & 32 & 0\end{array}$

36 ca0c0caa0babaacaacacabaaaaaaaaa0caaab0aa $60 \begin{array}{lllll}60 & 27 & 10 & 17 & 12\end{array}$

37 c000bcac0ccca0cca0acaaaaaabaaca0a0abb0ac $40 \quad 37 \quad 1027 \quad 22$

39 cccc0caa00abacbaaccca0aabaacabbacaaaa00b $42 \quad 42 \quad 15 \quad 27 \quad 15$

40 c0b0a0ca0accaacbacc0aa0caa0aabbbcaac0a0b $37 \quad 40 \quad 152522$

41 caabbcacaaaaaaaaacccaaaaaabaabaaaaabbbba $\begin{array}{llllll}65 & 35 & 20 & 15 & 0\end{array}$

42 aba0bc0abacbaacaabaccaaaacaaaaaacaa0abb0 $55 \quad 35 \quad 17 \quad 17 \quad 10$

43 0b0cabccb0cba0baabbc0abccccbbc0babbbaacc $20 \quad 65 \quad 35 \quad 45 \quad 0$

44 caabbc0ab000aacaacacacc0aa0aa0ba0aa0aa0b $45 \quad 30 \quad 12 \quad 17 \quad 25$

$\begin{array}{lllllll}45 & \text { abbb00ba0bbb0ac0ab0cacbc0a0ba0baacaaaaaa } & 37 & 40 & 27 & 12 & 22\end{array}$

47 aaabbccaaaaaaaaaaaaabaaaaaaabaaaaaabbb $\begin{array}{llllll}77 & 22 & 17 & 5 & 0\end{array}$

48 cb0babb000bbabbaabac0abcac00abbba00a0ccb $25 \quad 50 \quad 35 \quad 15 \quad 25$

$\begin{array}{llllllll}50 & \text { cb0b0cbbb00cb0caacaaacbaaa00aa0aaaaaabbb } 40 & 40 & 25 & 15 & 20\end{array}$

$\begin{array}{lllllll}51 & \text { cc0acaabbcccccaabaaaacaaaacaaabcbabccba } & 45 & 52 & 17 & 35 & 2\end{array}$

$\begin{array}{lllllll}52 & \text { abcbcababaaba0c0acccabbaaa0aabbaaa0aabba } & 47 & 42 & 27 & 15 & 10\end{array}$

$\begin{array}{lllllll}53 & \text { cabcbcbabbbc0bbaaccaaaaaabababb0c000bbca } & 32 & 55 & 35 & 20 & 12\end{array}$

$\begin{array}{lllllll}55 & \text { cb0c0ccc0accc0c00bacabac0caaac0a00a0a00c } & 25 & 42 & 7 & 35 & 32\end{array}$

$\begin{array}{lllllll}56 & \text { acbbbbaacacbbaaaaaaa0acbaaacabbaca00abaa } & 52 & 40 & 25 & 15 & 7\end{array}$

$\begin{array}{lllllll}57 & \text { ccbcacccbcbccbcac0aab0cbac00cbaacbaabcab } & 25 & 65 & 25 & 40 & 10\end{array}$

$\begin{array}{lllllll}58 & \text { 0b0bbcabbaabcc0aacaababaacacab0aa00bbcba } & 37 & 47 & 30 & 17 & 15\end{array}$

$\begin{array}{lllllll}59 & \text { cccbbcba0bbbbbcacbaaaacbaaababbaaa0ca0ba } & 37 & 55 & 35 & 20 & 7\end{array}$

$\begin{array}{lllllll}60 & \text { caabbbbaaacbbacaa0aaaaaaaaaaaaaaaaaacab } & 70 & 27 & 17 & 10 & 2\end{array}$

\section{Resultados del Postest de COMVda}

\begin{tabular}{|c|c|c|c|c|c|c|c|c|c|c|}
\hline I0 & $\mathrm{Nt}$ & P1 & P2 & Matriz de casos y variables & $\mathrm{Ia} \mathrm{Er} \mathrm{Ib}$ & $\mathrm{Ib}$ Ic & I0 & $\mathrm{Nt}$ & P1 & $\mathrm{P} 2$ \\
\hline 10 & 45 & 67 & 38 & aaabaacaaaabaaaaacaaabaaaaaaaabbcaaaabab & $\begin{array}{lll}75 & 25 & 17\end{array}$ & 7 & 0 & 63 & 76 & 63 \\
\hline 12 & 17 & -19 & -5 & cbcbbccacabbbccaaaaaabaabaacabaacaaaaccb & 475225 & 2527 & 0 & 22 & -24 & -11 \\
\hline 22 & 3 & -7 & -11 & abbbaccacabcbccaacbbaaabbcaaabcbcaabbacc & 376232 & 3230 & 0 & 7 & -24 & 07 \\
\hline 0 & 25 & 26 & 07 & bcccacbacaaaaabaabcaabaaaaaaaa)abaaaaabc & 623517 & 1717 & 2 & 45 & 26 & 63 \\
\hline 0 & 14 & -24 & 07 & ccbcacababbbcacaacaaaabbacacaaaaaaaabacb & 524722 & 2225 & 0 & 29 & -12 & 26 \\
\hline 50 & 13 & 12 & 00 & ab0b0abac0b0a0caab000cacaaa00b0a0a0a00bc & $\begin{array}{lll}32 & 30 & 17\end{array}$ & 1712 & 37 & 18 & 09 & -5 \\
\hline 0 & 59 & 38 & 82 & caabbaaaaabaccaaacaaabcaabaaaaaaaaacaa & $7227 \quad 12$ & 1215 & 0 & 59 & 38 & 82 \\
\hline 0 & -7 & 12 & -30 & caabbbbbbcbcbababbbcabbabaccabbbcaacacbc & 277247 & 4725 & 0 & -7 & 12 & -30 \\
\hline 22 & 22 & 21 & 13 & acccbaaabbabaacabcacab0acabaaa0accaaa0aa & $\begin{array}{lll}52 & 40 & 17\end{array}$ & 1722 & 7 & 33 & 30 & 07 \\
\hline 17 & 4 & -36 & 19 & cccbcccabaabcccacaaaaabaaaaacbaaabacbccb & 455520 & 2035 & 0 & 18 & -36 & 26 \\
\hline 7 & 22 & 26 & -24 & cabcbbabbcbcbabaabbaacaaaaaaabbacaaaaaac & 524730 & 3017 & 0 & 29 & 26 & -11 \\
\hline 7 & 25 & -44 & 69 & abbbccbaaaabccbaacaaabaccbaaaaabcaaaccbb & 475227 & 2725 & 0 & 22 & -49 & 63 \\
\hline 5 & 17 & -36 & 32 & abaaaaaaaaabbaabaaaaaacbaaabaaaacaaabbaa & 752520 & 5 & 0 & 63 & 51 & 44 \\
\hline 2 & 30 & 30 & 44 & ccbcbcbacaabaacaabcaaaaaaaaabbaaaaaacbb & $57 \quad 42 \quad 22$ & 2220 & 0 & 37 & 00 & 44 \\
\hline 10 & 8 & -15 & -36 & c0bbacbc0bbbcbbaabacaaaaabababbacacbb0aa & 375537 & 3717 & 7 & 10 & -15 & -24 \\
\hline 0 & 44 & 12 & 26 & ccccbcaaabbbcacaababaaaaaaaaaaaaaacbcaa & $\begin{array}{lll}60 & 40 & 17\end{array}$ & 1722 & 0 & 40 & -12 & 44 \\
\hline 10 & 53 & 38 & 69 & aabbbc0aa0abaaaaaba0aabaab0aa0baaaaaabac & 602722 & 5 & 12 & 47 & 26 & 56 \\
\hline 25 & 4 & -12 & -11 & c0bbacbb0bbbcbbaabacaa0bacb0abba0aabbcab & 305742 & 4215 & 12 & 2 & -19 & -11 \\
\hline 12 & 9 & -28 & -5 & cccbacbbabbbccbaabacacaababcabcacaaabbba & 376235 & 3527 & 0 & 7 & -24 & -11 \\
\hline 5 & 13 & -19 & -42 & aacbbcaabcccbccaaccaaabaaaababaacaabbcba & 475225 & 2527 & 0 & 22 & -24 & -30 \\
\hline 27 & 35 & 30 & 07 & ccabacbb0cbca00aabaaaaaaaa00a0baaaaaa0ba & $\begin{array}{lll}52 & 30 & 17\end{array}$ & 1712 & 17 & 38 & 26 & 07 \\
\hline 37 & 18 & 30 & -5 & ccacbaaabaabaacaacaaabacaaaaaabaacaabaac & 653515 & 1520 & 0 & 48 & 38 & 44 \\
\hline 12 & 5 & -15 & -5 & cccbacbbbbbbccbaabacaababa0bacaaaaaacaca & 425530 & 3025 & 2 & 15 & -12 & -11 \\
\hline 2 & 34 & 00 & 13 & caccbcaаaаabaccaacaаaаacaаaаaаbaаaаaаaаa & 7227 & 720 & 0 & 59 & 26 & 82 \\
\hline 15 & 14 & -19 & 19 & cbcbbcbbcaacacabccccabbcaaaaacaaabaabccc & 376225 & 2537 & 0 & 7 & -12 & 26 \\
\hline 7 & 44 & 55 & -5 & acacbaaacaabaacaabaaccaabaaaaabaacaaabac & 653515 & 1520 & 0 & 48 & 38 & 44 \\
\hline 2 & 12 & -12 & -5 & acabbcacaccbabcaabbcaabababacbbbbaabccca & 376235 & 3527 & 0 & 7 & -12 & 07 \\
\hline 5 & 5 & -7 & 07 & bbbbacabacbbcccaabccabbabbbaacaaabcaaaaa & 425735 & 3522 & 0 & 14 & 00 & 07 \\
\hline 0 & 22 & 12 & 07 & bccbacbbacaaaacaabccabaabaababbacbaabbba & 455535 & 3520 & 0 & 18 & 00 & 07 \\
\hline 12 & 39 & 26 & 32 & aaaaabbaabacaabaacaaaaaaaaaaaaaaaaabaa & $82 \quad 17 \quad 12$ & 125 & 0 & 74 & 63 & 63 \\
\hline 20 & 13 & -19 & 19 & ccbbacbaaabbcbcaacacacabaaaaabbbabaaaabb & 475232 & 3220 & 0 & 22 & 00 & 26 \\
\hline 0 & 10 & -36 & -49 & cbbbbcbbcccbaccaacaaabaaaaabacaabaaabbbb & 425735 & 3522 & 0 & 14 & -24 & -30 \\
\hline 12 & 47 & 26 & 32 & ccccbcaaababaccaacacaaaabaaaaaaaaaab0aa & $6235 \quad 12$ & 1222 & 2 & 45 & -7 & 63 \\
\hline 22 & 22 & 09 & 00 & cbabbcaaacbbabcca0aaaabbaaaaaabbaaaaa0cc & $5242 \quad 25$ & 2517 & 5 & 32 & 05 & 44 \\
\hline 15 & 22 & 00 & 00 & accbbcaaa0abaabaaccaaaaaaaaaaabacaaaaa0b & 653015 & 1515 & 5 & 50 & 17 & 51 \\
\hline 22 & 18 & 34 & 13 & cbbbbbcaaaabaacaaccaaaaaaaaaabbaaaaaabb & 623725 & 2512 & 0 & 44 & 12 & 82 \\
\hline 0 & 48 & 26 & 82 & ccccacacaabbaaaaaccaabaabaaaaaaaaaabaaa & $6732 \quad 12$ & 1220 & 0 & 52 & 38 & 63 \\
\hline 10 & 38 & 05 & 26 & ac00bcaabacb0acaabaccaacaaaaaaaacaaaabbb & 553717 & 1725 & 2 & 37 & 00 & 26 \\
\hline 0 & -11 & 09 & -24 & aaacacabbacbbcaaacacaaaababaaabaabaabacc & $57 \quad 4222$ & 2220 & 0 & 37 & 26 & 26 \\
\hline 25 & 30 & 42 & 19 & aaabacaabcabaabaacacaccbaaaaaabaaaaaaaab & 673217 & 1715 & 0 & 52 & 63 & 44 \\
\hline 22 & 18 & 26 & -17 & ccccacba0bbbbac0abacaa00aa0ba0baacaaaaaa & $45 \quad 4020$ & 2020 & 15 & 25 & 26 & -17 \\
\hline 0 & 67 & 38 & 99 & aaabaabaaaaaaaaaaaaabaaaaaaabaaaaaabaa & $87 \quad 12 \quad 12$ & 12 & 0 & 82 & 76 & 99 \\
\hline 25 & 0 & -15 & -5 & ccccbcbcabbbabcaabacaabcaca0aabba0aabcca & 375727 & 2730 & 5 & 9 & -36 & 19 \\
\hline 20 & 20 & -12 & 26 & cbbbbcbbbaccbbcaacaaacbaaabaaaaaaaaaabbb & 475235 & 3517 & 0 & 22 & -24 & 44 \\
\hline 2 & 19 & -7 & -30 & cc0acaabbccccccaabaaaaaaaaaaaaaababbcba & $\begin{array}{lll}55 & 42 & 17\end{array}$ & 1725 & 2 & 34 & -7 & 07 \\
\hline 10 & 27 & 05 & 44 & abccacbabaaaaaaacccabbaaa0aabaaaaaaabba & 623520 & 2015 & 2 & 45 & 26 & 63 \\
\hline 12 & 5 & -32 & -42 & ccccacbabbbcacbaaccaaaaabaababbbcaaabbca & 425730 & 3027 & 0 & 14 & -12 & -30 \\
\hline 32 & 4 & -15 & 07 & cbbbacbbabbbcbbaabacabacacaaacbaaaaaacbc & 425735 & 3522 & 0 & 14 & -24 & 26 \\
\hline 7 & 33 & 12 & -11 & aaabbabacacabaaaaaaaaaaaaaacabbaba0aabaa & 702720 & 20 & 2 & 57 & 51 & 07 \\
\hline 10 & -6 & -24 & -42 & cbabacaabcbbbb0cccabaacbac0baaaaababbcaa & 405532 & 3222 & 5 & 13 & -7 & -11 \\
\hline 15 & 14 & -40 & 13 & aacbbccabaababbaabaababaaaaaabbaaa0bbcba & 524535 & 3510 & 2 & 30 & -12 & 44 \\
\hline 7 & 10 & -19 & -5 & cacbaaaabbabbbcaabaaaaaaaaababbaaaacabba & 604030 & 3010 & 0 & 40 & 12 & 07 \\
\hline 2 & 57 & 26 & 63 & caabbbabbacaaaaaabaaaaaaaaaaaaaaaaabab & 752520 & 20 & 0 & 63 & 51 & 63 \\
\hline & $1,1,1$ & 3,4 & 9,9 & Medias (Nt, P1 y P2 del postest) & & & & 32,6 & $8, I$ & 2,61 \\
\hline
\end{tabular}

Medias (Nt, P1 y P2 del pretest) 
Del mismo modo, para analizar los progresos organicistas, se deben utilizar tan sólo las notas del parcial 2. En este caso, la comparación de las mismas entre pretest y postest (columnas P2 del pretest y del postest de la tabla 5) mediante la prueba $T$ para muestras relacionadas, proporciona una diferencia de medias de 16,21; significativa al $99 \%$ de confianza.

Como se puede apreciar, se dan mayores logros organicistas que constructivistas, poniendo de manifiesto que los alumnos abandonan sus posiciones reduccionistas de manera más fácil o rápida de la que aprenden a diferenciarlas de las posiciones específicamente constructivistas.

c) Otras opciones de análisis son posibles, tales como analizar el porcentaje de estudiantes constructivistas frente a los que son más racionalistas, y frente a los que son más empiristas. Lo primero se infiere de la comparación de los índices Ia e Ib y lo segundo de la comparación de Ia e Ic. La exposición de estos resultados excede de los límites de extensión de este trabajo.

\section{CONCLUSIONES}

\section{A. Sobre el cuestionario}

El cuestionario construido para evaluar el aprendizaje y el conocimiento del estudiante está formado, como se ve en el anexo 1, por 40 ítems y tres opciones de respuesta cada uno. El análisis de la bondad de los ítems permite concluir que, de los 40 ítems, tan sólo tres son de baja calidad frente a los trece que se encontraron en el cuestionario sobre visión de Ciencias (Marín y Benarroch, 2009). Asimismo, la correlación entre índices en el cuestionario (ver parte inferior de la tabla 4) tal y como se presenta en el anexo I ofrece una imagen estable del producto actual.

Si el cuestionario en su estado actual es válido y fiable, también es mejorable (por ejemplo, tratando de modificar los tres ítems de calidad inferior). Por tanto, creemos que lo más importante no es el cuestionario en su estado actual, sino la metodología puesta en juego para evaluar la bondad de los ítems, que, aplicada sistemáticamente, permite disponer de un cuestionario cada vez más válido y fiable.

\section{B. Sobre el contenido VdA y la formación de profe- sores de ciencias}

Respecto a la utilización del cuestionario COMVdA en un contexto de enseñanza, se constata que, a pesar de los bajos rendimientos obtenidos en el pretest, tras el breve período de tiempo de enseñanza, la situación inicial cambia, y se obtienen incrementos significativos en los postest, y sólo algo inferiores a los que se obtuvieron para la visión de ciencias $(\mathrm{VdC})$.

Esto tiene una lectura motivadora para la formación docente y sugiere que si se da un espacio a la enseñanza de $\mathrm{VdA}$ semejante a $\mathrm{VdC}$, con estrategias didácticas semejantes, el alumno puede aprender cuestiones generales sobre aprendizaje y epistemología del conocimiento a la par que las relacionadas con la epistemología de la ciencia.

Sobre esto habría que recordar que algunos autores defienden que no es posible un cambio epistemológico sobre el conocimiento de ciencias (ni del alumno) si esto no conlleva también un cambio sobre posiciones epistemológicas generales (Delval, 1997; Pozo et al., 1996).

Además de las cualidades de COMVdA para fomentar el cambio sobre VdA, su diseño también permite precisiones en la evaluación del alumnado. Así, permite analizar de forma diferenciada los rendimientos para ítems tipo C3 y $\mathrm{O} 3$ arrojando datos sobre el progreso «constructivista» y sobre el abandono de posiciones reduccionistas.

En la actualidad, se continúa con el proceso de validación o falsación de ítems, y se trata de realizar una evaluación externa por expertos tanto de la sistemática de contextos como de la coherencia entre éstos y los ítems. Para ello, se está desarrollando una versión del método Delphi (Landeta, 1999) para Internet, cuyo detalle excede los límites de extensión de este trabajo.

\section{REFERENCIAS BIBLIOGRÁFICAS}

BAENA, M.D. (2000). Pensamiento y acción en la enseñanza de las ciencias. Enseñanza de las Ciencias, 18(2), pp. 217-226.

BOULTON-LEWIS, G.M., SMITH, D., MCCRINDLE, A.R., BURNETT, P. C. y CAMPBELL, K.J. (2001). Secondary teachers' conceptions of teaching and learning. Learning and Instruction, 11(1), pp. 35-51.

CASTILlA DEL PINO, C. (2000). Teoría de los Sentimientos. Barcelona: Tusquets.

DELVAL, J. (1997). Tesis sobre el constructivismo, en M.J. Rodrigo y J. Arnay (eds.). La construcción del conocimiento escolar (pp. 15-24). Barcelona: Paidós.
DESAULTES, S. (1993). La formation à l'enseignement des sciences: Le virage épistemologique. Didaskalia, 1, pp. 49-67.

DILLON, D.R., O'BRIEN, D.G., MOJE, E.B. y STEWART, R.A. (1994). Literacy learning in secondary school science classrooms: A cross-case analysis of three qualitative studies. Journal of Research in Science Teaching, 31(4), pp. 345-362.

FERNÁNDEZ, I., GIL, D., CARRASCOSA, J., CACHAPUZ, A. y PRAIA, J. (2002). Visiones deformadas de la ciencia transmitidas por la enseñanza. Enseñanza de las Ciencias, 20(3), pp. 477-488.

GARCÍA MADRUGA, J.A. (1990). Aprendizaje por descubrimiento frente a aprendizaje por recepción. La teoría del 
aprendizaje verbal significativo, en C. Coll, J. Palacios y A. Marchesi (eds.). Desarrollo psicológico y educación, II (pp. 81-93). Madrid: Alianza.

HEWSON, P.W. y HOLLON, R. (1994). Connecting thought and action in high school science classrooms. WisconsinMadison: Wisconsin Center for Education Research.

HEWSON, P.W., KERBY, H.W. y COOK, P.A. (1995). Determining the conceptions of teaching science held by experienced high school science teachers. Journal of Research in Science Teaching, 32(5), pp. 503-520.

HOLLON, R. y ANDERSON, C. (1987). Teacher's beliefs about student's learning processes in science: Self-reinforcing beliefs sistems. Artículo presentado en The Annual Meeting of A.E.R.A. Washington D.C.

HUIBRETSE, I., KORTHAGEN, F. y WUBBELS, T. (1994). Physics teachers' conceptions of learning, teaching and professional development. International Journal of Science Education, 16(5), pp. 539-561.

LANDETA, J. (1999). El método delphi. una técnica de previsión para la incertidumbre. Barcelona: Ariel.

LÓPEZ, J.I. (1995). El conocimiento profesional de los profesores acerca de las concepciones de los alumnos. Dos estudios de caso en la enseñanza y aprendizaje de las ciencias. Tesis doctoral inédita. Universidad de Sevilla.

LORSBACH, A.W., TOBIN, K., BRISCOE, C. y LAMASTER, S. U. (1992). An interpretation of assessment methods in middle school science. International Journal of Science Education, 14(2), pp. 305-317.

LUFFIEGO, M. (2001). Reconstruyendo el constructivismo: Hacia un modelo evolucionista del aprendizaje de conceptos. Enseñanza de las Ciencias, 19(3), pp. 377-392.

MARÍN, N. (2003a). Conocimientos que interaccionan en la enseñanza de las ciencias. Enseñanza de las Ciencias, 21(1), pp. 65-78.

MARÍN, N. (2003b). Visión constructivista dinámica para la enseñanza de las ciencias. Enseñanza de la Ciencias, Extra, pp. 43-55.

MARÍN, N. (2005). La enseñanza de las ciencias en Educación Infantil. Grupo Editorial Universitario: Granada.

MARÍN, N. y BENARROCH, A. (2009). Desarrollo, validación y evaluación de un cuestionario de opciones múltiples para identificar y caracterizar las visiones sobre la ciencia de profesores en formación. Enseñanza de las Ciencias, 27(1), pp. 89-108.

MATTHEWS, M. (1994).Science Teaching; The Role of History and Philosophy of Science, Routledge, New York, NY.

MCCOMAS, W. F., CLOUGH, M. P. y ALMAZROA, H. (1998). The role and character of the nature of science in science education, en McComas, W.F. (ed.). The nature of science in science education. rationales and strategies (pp. 3-39). Dordrecht, The Netherlands: Kluwer Academic Publishers.

MELLADO, V. (1996). Concepciones y prácticas de aula de profesores de ciencias, en formación inicial, de primaria y secundaria. Enseñanza de las Ciencias, 14(3), pp. 398-402.

OSBORNE, J., COLLINS, S., RATCLIFFE, M., MILLAR, R. y DUSCHL, R. (2003). What «Ideas-about-science» should be taught in school science? A delphi study of the expert community. Journal of Research in Science Teaching, 40(7), pp. 692-720.

PEÑALVER, C. (1988). El pensamiento sistémico: del constructivismo a la complejidad. Investigación en la Escuela, 5, pp. 11-16.

PÉREZ ECHEVERRÍA, M.P., POZO, J.I. y RODRÍGUEZ, B. (2003). Concepciones de los estudiantes universitarios sobre el aprendizaje, en Monereo, C. y Pozo, J.I. (coords). La universidad ante la nueva cultura educativa: enseñar y aprender para la autonomía, pp. 33-44. Madrid: Síntesis.

PIAGET, J. (1977). Epistemología genética. Solpin: Argentina. (Ver. Orig. L'epistemologie gènetique. Presses Universitaires de France. París. 1970).

PORLÁN, R. (1989). Teoría del conocimiento, teoría de la enseñanza y desarrollo profesional. Las concepciones epistemológicas de los profesores. Tesis doctoral inédita. Universidad de Sevilla.

PORLÁN, R. y MARTÍN DEL POZO, R. (2004). The conceptions of in-service and prospective primary school teachers about the teaching and learning of science. Journal of Science Teacher Education, 15(1), pp. 39-62.

PORLÁN, R., RIVERO, A. y MARTÍN DEL POZO, R. (1997). Conocimiento profesional y epistemológico de los profesores I: Teoría, métodos e instrumentos. Enseñanza de las Ciencias, 15(2), pp. 155-164.

PORLÁN, R., RIVERO, A. y MARTÍN DEL POZO, R. (1998). Conocimiento profesional y epistemología de los profesores II: Estudios empíricos y conclusiones. Enseñanza de las Ciencias, 16(2), 271-288.

POZO, J.I. (1989). Teorías cognitivas del aprendizaje. Madrid: Morata.

POZO, J.I. (2003). Adquisición del conocimiento. Cuando la carne se hace verbo. Madrid: Morata.

POZO, J.I. y SCHEUER, N. (1999). Las concepciones sobre el aprendizaje como teorías implícitas, en Pozo, J.I. y Monereo, C. (eds.). El aprendizaje estratégico (pp. 87-108). Madrid: Aula XXI/Santillana.

POZO, J.I., SCHEUER, N., PÉREZ ECHEVERRÍA, M. P., MATEOS, M. y DE LA CRUZ, M. (2006). Nuevas formas de pensar la enseñanza y el aprendizaje. Barcelona: Graó.

PRAWAT, R.S. (1992). Teachers' beliefs about teaching and learning: A constructivist perspective. American Journal of Education, 100(3), pp. 354-395.

RUIZ, C., PORLÁN, R., DA SILVA, C. y MELLADO, V. (2005). Construcción de mapas cognitivos a partir del cuestionario INPECIP. Aplicación al estudio de la evolución de las concepciones de una profesora de secundaria entre 1993 y 2002. Revista Electrónica de Enseñanza de las Ciencias, 4(1). Retrieved from http:/www.saum.uvigo.es/reec.

SANMARTÍ, N. (2002). Didáctica de las ciencias en la Educación Secundaria Obligatoria. Madrid: Síntesis.

VÁZQUEZ, A., ACEVEDO, J. A. y MANASSERO, M. A. (2004). Consensos sobre la naturaleza de la ciencia: evidencias e implicaciones para su enseñanza. Revista Iberoamericana de Educación, edición digital. En http://www.rieoei. org/deloslectores/702Vazquez.PDF. 
1. Imaginemos que podemos pesar todo lo que sabe una persona adulta y todo lo que existe en el medio ¿cuánto pesa lo $1^{\circ}$. en relación con lo $2^{\circ}$.?

a) Menos, siempre se va aprendiendo del medio.

b) No es posible comparar, pues son cosas diferentes.

c) Básicamente menos pero más considerando la imaginación.

2. La imagen que el sujeto tiene de una silla es como:

a) Una fotografía de la silla más o menos distorsionada.

b) Una copia parcial de la silla cada vez más completa.

c) Una idea útil pero no se sabe si es o no imagen de la silla.

3. La precisión de los tenistas ajustando cada vez más las bolas a las líneas del campo hace pensar que construimos una idea de la realidad que:

a) La distorsiona cada vez menos.

b) Es copia cada vez más completa.

c) Es cada vez más útil.

4. El dicho «nada es verdad ni mentira, todo depende del cristal con que se mira» se parece más a la afirmación $(\mathrm{OC}=$ objeto conocido; $\mathrm{OR}=$ objeto real):

a) OC es copia parcial de OR que varía de un sujeto a otro.

b) OC no es la imagen de OR, así que nada es verdad ni mentira.

c) OC es la imagen de OR filtrada por la lente del sujeto.

5. Algunos afirman que existe una realidad construida por el sujeto que es diferente a la realidad externa al sujeto. ¿Te lo crees?

a) Sí, hasta el punto que no se puede conocer cuál es la diferencia.

b) Sí hay diferencias, pero también notables correspondencias.

c) No hay diferencias. Puede que la realidad construida sea parcial, pero toda se corresponde con la real.

6. Conforme aprendo más de un objeto, ¿es adecuado afirmar que su imagen mejora?

a) Sí, una imagen menos distorsionada aunque siempre será difusa.

b) Sí, una imagen más completa y por tanto cada vez más nítida.

c) No, sólo sirve para usarlo con nuevas posibilidades.

7. Sobre el mundo cotidiano que conocemos, ¿es posible que un mayor conocimiento incorpore nuevos objetos tan reales como pueda ser una silla?

a) Sí, ocurre cuando se estudia en detalle cualquier cosa.

b) No, lo que lleva es a conocer más los objetos o ver nuevas relaciones.

c) No, lo que supone es tener imágenes más completas de los objetos.

8. Un cambio sustancial en mi conocimiento de la realidad:

a) Cambiará mi modo de afrontar la realidad.

b) Hará que conozca de un modo más preciso la realidad.

c) Me permitirá conocer más la verdad de la realidad.

9. Simplificando, la organización del conocimiento del sujeto se parece más a:

a) Diversos tipos de memorias interconectadas. b) Elementos orgánicos relacionados como en cualquier ser vivo.

c) Una estructura conceptual jerarquizada.

10. La relación entre las partes que integran el conocimiento es tal que su suma:

a) Es el todo, como el encaje de las piezas de un reloj.

b) Es el todo, como el encaje de las piezas de un puzle.

c) Es menos que el todo, como los órganos de un ser vivo.

11. El conocimiento ¿se puede estudiar por las partes que lo componen?

a) No, la capacidad de las partes combinadas es mayor que separadas.

b) Sí, como máquina desmontable, se pueden analizar partes y relaciones.

c) Sí, como puzle de asociación de ideas se puede analizar por partes.

12. Viendo el conocimiento como una construcción, el sujeto construye:

a) Con ladrillos y cemento del medio que él va colocando ordenadamente.

b) Desde dentro, el medio sólo fomenta o restringe.

c) Con ladrillos y cemento del medio y antes de colocar retoca o desecha.

13. Entre los diferentes contenidos del conocimiento, ¿existe alguno que sea más objetivo o verdadero que los demás?

a) No, todo es subjetivo, mejor hablar de utilidad que de verdad.

b) Sí, los contenidos matemáticos encajan con exactitud.

c) Sí, los contenidos construidos por la experiencia.

14. ¿Puede llegar a ser OC un conocimiento verdadero de OR?

a) Sí, con esfuerzo se van quitando distorsiones de OC.

b) Sí, es posible ir completando OC.

c) No, como mucho, cada vez más útil.

15. ¿Cómo aparecen las primeras construcciones cognitivas en el sujeto?

a) Las aporta la herencia y se van llenando con la experiencia.

b) Al tomarlas del medio a través de la experiencia.

c) «Digiriendo» nuestro cuerpo las experiencias con el medio.

16. La mayor parte del significado que damos a «velocidad» se ha tomado de:

a) Conversaciones con los demás sobre este concepto.

b) La usual explicación en el entorno académico.

c) Experiencias en bici, al cruzar la calle o jugar al tenis.

17. Un niño se ha enfrentado en múltiples ocasiones a problemas donde la solución conlleva hacer palanca. ¿Qué le aporta estas experiencias?

a) Destrezas e ideas sobre el funcionamiento de las palancas.

b) La ley que existe entre brazo y fuerza de la palanca.

c) Ideas semejantes al contenido académico sobre palancas.

18. Principalmente, además de la ayuda de los mayores, ¿qué es lo que más influye para aprender a andar?

a) Impulso de imitar a los mayores.

b) Evitar golpes de las caídas.

c) Instinto heredado para andar. 
19. Principalmente, ¿qué es lo que más te ha influido para saber el momento de cruzar la calle en función de la rapidez de los coches?
a) El consejo de los mayores
b) La experiencia personal.
c) La intuición hereditaria.

20. ¿Qué te influyó más para aprender a contar objetos?

a) Acciones como ordenar, dar, agrupar o separar objetos.

b) La experiencia con el orden natural de los objetos.

c) Las instrucciones dadas por mayores y maestros.

21. ¿Qué te influyó más para aprender a encontrar tus objetos favoritos?

a) La estructura espacial dada de forma hereditaria

b) Imitando a los mayores viendo cómo ellos los encuentran

c) La motivación por encontrarlo y vivencias recuperando objetos

22. En general, ¿hay diferencias entre lo que se adquiere por vivencia personal y por información?

a) Sí, lo primero da más habilidades; lo segundo da más ideas.

b) Sí, el grado de valor y utilidad que tienen para el sujeto.

c) No, todo se va añadiendo en un entramado de ideas.

23. Todo lo que se aprende ¿se puede expresar verbalmente?

a) No, parte sólo sirve para tener habilidades.

b) Depende, sólo cuando está bien integrado.

c) Sí, todo lo que se aprende se puede decir.

24. ¿Podrá el instructor de tenis explicar todas sus habilidades de jugador a los novatos?

a) Depende, en algunos casos, habilidades y expresión verbal coinciden.

b) No, parte sólo la podrá expresar con gestos y ademanes

c) Sí, con el tiempo podrá explicar todas sus habilidades.

25. ¿Es cierto que «todo depende del cristal con que se mira»?

a) Todo depende del tipo de objeto que se perciba.

b) Sí, todo lo que se percibe es interpretado.

c) Sí, los sentidos filtran como un cristal todo lo que se percibe.

26. ¿Es cierto que en ocasiones se ve lo que queremos ver?

a) No, las cosas se ven como son otra cosa es que queramos ver otra cosa.

b) Sí, el significado que damos a lo que vemos es un asunto también afectivo.

c) Sí, la imagen distorsionada invita a ver algo diferente.

27. La mayor parte de los significados que tiene un niño del fútbol provienen de:

a) Sus vivencias de los partidos que ha jugado.

b) Las charlas con sus compañeros sobre fútbol.

c) Las explicaciones de su instructor escolar.

28. Aprender se parece a:
a) Filtrar para separar una mezcla
b) Escribir sobre folio en blanco.
c) Digerir la comida.

29. Sobre cualquier objeto ¿siempre se puede aprender algo más?

a) No, sólo sería cierto para objetos complejos.

b) Depende, hay objetos de los que se posee una imagen completa.

c) Sí, nuevas experiencias pueden aportar más información.
30. Lo que se aprende nuevo, ¿cómo llega a formar parte del conocimiento que el sujeto ya posee?

a) Reorganizando lo que ya posee para acomodar lo nuevo.

b) Relacionando lo nuevo con algunos que ya posee.

c) Añadiendo lo nuevo a lo que ya posee.

31. Aprende de memoria una fórmula que no sabe usar para resolver un problema. La primera razón que explica esto es:

a) No sabe relacionarla con los datos del problema.

b) El problema no admite el uso mecánico de la fórmula.

c) Para usarla hay al menos que comprenderla.

32. ¿Qué factor determina más que sea tan difícil olvidar a montar en bicicleta y tan fácil olvidar el número de un teléfono?

a) El grado de motivación del sujeto en aprender.

b) El $n^{\circ}$. de teléfono no se olvida si se emplea el mismo tiempo.

c) El modo de incorporarse el nuevo conocimiento.

33. Lo que percibimos a través de los sentidos ¿es conocimiento?

a) No, haría falta digerirlo o cuando menos darle significado.

b) Casi, pues antes sería filtrado.

c) Sí, son las piezas de base del conocimiento.

34. El refrán «para aprender, perder» quiere decir que aprender algo para que no se olvide requiere:

a) Gastar mucho tiempo en el aprendizaje.

b) Incorporar lo nuevo sustituyendo lo viejo.

c) Equivocaciones, contradicciones, errores, etc.

35. ¿Es cierto el refrán «quien desea aprender, pronto llegará a saber»?:

a) Sí, la necesidad es primordial para aprender sin olvidar.

b) Sí, pero sería un tipo de saber que se olvida fácilmente

c) No, para ser cierto la persona debe tener cierto nivel de estudios.

36. En general, lo aprendido por vivencia personal con poca o ninguna ayuda de los demás, respecto a lo aprendido por información verbal, es más:

a) Sencillo y lógico de aprender.

b) Fácil y convincente de comunicar.

c) Lento de aprender y más duradero.

37. Las cosas ¿las ven nuestros sentidos como son?

a) No se puede saber si lo que se ve es lo que parece ser.

b) En parte, cuanto más se estudien más se ven como son.

c) En muchos casos sí, en otros hay que volver a mirar mejor.

38. Los colores que percibe el sujeto de los lápices de una caja:

a) Los aportan directamente los lápices.

b) Sólo son un producto de tu cuerpo.

c) Están distorsionados pero existe relación.

39. La imagen de un objeto es filtrada primero con los sentidos y después por nuestro conocimiento previo, por eso es mejor pensar que lo que creemos que es el objeto:

a) Es sólo un modelo útil para manejar el objeto.

b) Es la imagen del objeto aunque filtrada o distorsionada.

c) Es el objeto, al menos una imagen parcial.

40. Haciendo «pesas» se desarrollan los músculos. ¿Se desarrolla el conocimiento de forma semejante?

a) No, músculo y conocimiento crecen de modo diferente.

b) Sí, pero la relación no es tan directa.

c) Sí, a más ejercicio más conocimiento. 


\title{
Questionnaire of multiple-choice to assessment beliefs about learning of science
}

\author{
Marín, Nicolás ${ }^{1}$ y Benarroch, Alicia ${ }^{2}$ \\ ${ }^{1}$ Departamento de Didáctica de las Matemáticas y de las Ciencias Experimentales. Universidad de Almería \\ ${ }^{2}$ Departamento de Didáctica de las Ciencias Experimentales. Universidad de Granada \\ nicolas.marin@gmail.com \\ aliciabb@ugr.es
}

\section{Summary}

This paper shows the design of a multiple-choice questionnaire (COMVdA) to assess the beliefs related to science learning in the context of the training of future teachers of secondary schools. It has tried to maintain a principle of maximum coherence with the theoretical and structural criteria that have been previously used in the design, development and evaluation of a previous questionnaires about the vision of science (COMVdC).

The main feature of the design of the questionnaire is that we have tried to fit it to the content of the science learning that we should taught to future teachers of secondary schools. This is, after doing a literature review on the beliefs related to science learning, we conclude the desirability of a new questionnaire that could be done in a hour, in the context of teacher training at secondary school. So, a pre-postest administration may reveal the degree of learning related to the beliefs on the science learning.

Specifically, this study is aimed to:

a) Show the contents, topics and issues related to science learning and its relationship to teaching (which we call "systematic contexts") that might be of interest for future science teachers in secondary and that, accordingly, we are trying to evaluate with the questionnaire.

b) Describe the process and illustrate the development of the initial multiple-choice items, based on the previously established systematic contexts. Using the constructivist vision to make the choice to play the role of technically "correct" option and more empirical, rationalist, reductionist or realistic to specify the distractors.

c) Show in detail the process used in the validation of the initial questionnaire after their administration to a sample of 45 high school teachers in a course of the CAP (developed in 2003).

d) Discuss the usefulness and limitations of the final questionnaire, after administration to a second sample of 50 high school teachers in a course of the CAP developed in 2005. The main goal in 2003 was the purification and validation of items, but in 2005, the special emphasis was on the usefulness of the questionnaire for the assessment of teaching experience. In 2005, therefore, the questionnaire is used as the pretest and postest. Another important difference between the two years is that the criteria for analyzing the validity of each item were more accurate both, quantitatively and consensualy than those adopted in 2003.

e) Draw conclusions regarding the results of the questionnaire and its potential to be introduced as a tool for both assessment and instruction in the training of future science teachers.

In this paper, we show the questionnaire administered in 2005. It consists of 40 multiple choice items, of which only three items are of low quality. Therefore, it would be more correct for you exclude them in possible further analysis, for example, to calculate the differences between pretest and postest. This analysis should be undertaken only with items of upper and middle quality, which have been more valid and discriminatory.

Reliability is analised through the correlations between the indicators in the 2003 and 2005 tests, and the correlations between the indicators of pretest and postest in 2005. These correlations indicate the degree of stability or reliability of the items to give the same results to samples or to different moments of learning. The paper shows that all correlations between similar rates, with few exceptions that we discuss, are significant at least at $95 \%$ confidence.

In general, the COMVdA and the mechanics of selfapplied make up a system that is converging toward a growing valid and reliable questionnaire.

The COMVdA has very good qualities to be applied in educational contexts, such as the ability to be administered within an hour and be integrated into the teaching process as an effective teaching tool. Specifically, when we used COMVdA as a tool for assessment in 2005, we found that, despite the low marks obtained in the pretest, after the brief period of 10 hours of instruction, the situation changes and you get significant increases in the postest.

This is encouraging for the training of teachers because it suggests that if we teach $\mathrm{VdA}$ with similar time and teaching strategies respect to $\mathrm{VdC}$, students can learn about learning of science to the same extent as epistemology of science. 